\title{
AN ASYMPTOTIC ANALYSIS OF THE MEAN FIRST PASSAGE TIME FOR NARROW ESCAPE PROBLEMS: PART II: THE SPHERE*
}

\author{
ALEXEI F. CHEVIAKOV ${ }^{\dagger}$, MICHAEL J. WARD ${ }^{\ddagger}$, AND RONNY STRAUBE $\$$
}

\begin{abstract}
The mean first passage time (MFPT) is calculated for a Brownian particle in a spherical domain in $\mathbb{R}^{3}$ that contains $N$ small nonoverlapping absorbing windows, or traps, on its boundary. For the unit sphere, the method of matched asymptotic expansions is used to derive an explicit three-term asymptotic expansion for the MFPT for the case of $N$ small locally circular absorbing windows. The third term in this expansion, not previously calculated, depends explicitly on the spatial configuration of the absorbing windows on the boundary of the sphere. The threeterm asymptotic expansion for the average MFPT is shown to be in very close agreement with full numerical results. The average MFPT is shown to be minimized for trap configurations that minimize a certain discrete variational problem. This variational problem is closely related to the well-known optimization problem of determining the minimum energy configuration for $N$ repelling point charges on the unit sphere. Numerical results, based on global optimization methods, are given for both the optimum discrete energy and the arrangements of the centers $\left\{x_{1}, \ldots, x_{N}\right\}$ of $N$ circular traps on the boundary of the sphere. A scaling law for the optimum discrete energy, valid for $N \gg 1$, is also derived.
\end{abstract}

Key words. narrow escape, mean first passage time, matched asymptotic expansions, surface Neumann Green's functions, discrete variational problem, logarithmic switchback terms

AMS subject classifications. 35B25, 35C20, 35P15, 35J05, 35J08

DOI. $10.1137 / 100782620$

1. Introduction. The narrow escape problem concerns the motion of a Brownian particle confined in a bounded domain $\Omega \in \mathbb{R}^{d}(d=2,3)$ whose boundary $\partial \Omega=\partial \Omega_{r} \cup \partial \Omega_{a}$ is almost entirely reflecting $\left(\partial \Omega_{r}\right)$, except for small absorbing windows, or traps, labeled collectively by $\partial \Omega_{a}$, through which the particle can escape. Denoting the trajectory of the Brownian particle by $X(t)$, the mean first passage time (MFPT) $v(x)$ is defined as the expectation value of the time $\tau$ taken for the Brownian particle to become absorbed somewhere in $\partial \Omega_{a}$ starting initially from $X(0)=x \in \Omega$, so that $v(x)=E[\tau \mid X(0)=x]$. The calculation of $v(x)$ becomes a narrow escape problem in the limit when the measure of the absorbing set $\left|\partial \Omega_{a}\right|=\mathcal{O}\left(\varepsilon^{d-1}\right)$ is asymptotically small, where $0<\varepsilon \ll 1$ measures the dimensionless radius of an absorbing window. Since the MFPT diverges as $\varepsilon \rightarrow 0$, the calculation of the MFPT $v(x)$ constitutes a singular perturbation problem.

The narrow escape problem has many applications in biophysical modeling (see $[2],[16],[19],[39]$ and the references therein). For the case of a two-dimensional domain, the narrow escape problem has been studied with a variety of analytical methods in [19], [42], [43], [20], and Part I of this paper [30]. In this paper, we use

* Received by the editors August 15, 2009; accepted for publication (in revised form) January 15, 2010; published electronically March 26, 2010.

http://www.siam.org/journals/mms/8-3/78262.html

$\dagger^{\dagger}$ Department of Mathematics and Statistics, University of Saskatchewan, Saskatoon, SK, S7N 5E6, Canada (cheviakov@math.usask.ca). This author's work was supported by the NSERC (Canada).

${ }^{\ddagger}$ Department of Mathematics, University of British Columbia, Vancouver, BC, V6T 1Z2, Canada (ward@math.ubc.ca). This author's work was supported by the NSERC (Canada).

$\S$ Max-Planck Institute for Dynamics of Complex Technical Systems, 39106 Magdeburg, Germany (rstraube@mpi-magdeburg.mpg.de). This author's work was supported by the Ministry of Education of Saxony-Anhalt within the Research Center "Dynamic Systems."

836 
the method of matched asymptotic expansions to study the narrow escape problem in a certain three-dimensional context.

In a three-dimensional bounded domain $\Omega$, it is well known (cf. [19], [35], [38]) that the MFPT $v(x)$ satisfies a Poisson equation with mixed Dirichlet-Neumann boundary conditions, formulated as

$$
\begin{gathered}
\triangle v=-\frac{1}{D}, \quad x \in \Omega, \\
x \in \partial \Omega_{a}=\bigcup_{j=1}^{N} \partial \Omega_{\varepsilon_{j}}, \quad j=1, \ldots, N ; \quad \partial_{n} v=0, \quad x \in \partial \Omega_{r} .
\end{gathered}
$$

Here $D$ is the diffusivity of the underlying Brownian motion, and the absorbing set consists of $N$ small disjoint absorbing windows, or traps, $\partial \Omega_{\varepsilon_{j}}$ for $j=1, \ldots, N$ each of area $\left|\partial \Omega_{\varepsilon_{j}}\right|=\mathcal{O}\left(\varepsilon^{2}\right)$. We assume that $\partial \Omega_{\varepsilon_{j}} \rightarrow x_{j}$ as $\varepsilon \rightarrow 0$ for $j=1, \ldots, N$ and that the traps are well separated in the sense that $\left|x_{i}-x_{j}\right|=\mathcal{O}(1)$ for all $i \neq j$. With respect to a uniform distribution of initial points $x \in \Omega$ for the Brownian walk, the average MFPT, denoted by $\bar{v}$, is defined by

$$
\bar{v}=\chi \equiv \frac{1}{|\Omega|} \int_{\Omega} v(x) d x,
$$

where $|\Omega|$ is the volume of $\Omega$. The geometry of a confining sphere with traps on its boundary is depicted in Figure 1.1.

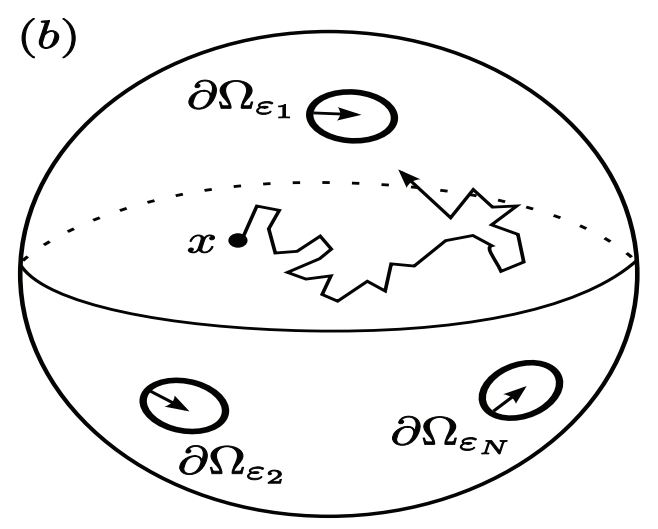

FIG. 1.1. Sketch of a Brownian trajectory in the unit sphere in $\mathbb{R}^{3}$ with absorbing windows on the boundary.

There are only a few results for the MFPT, defined by (1.1), for a bounded threedimensional domain. For the case of one locally circular absorbing window of radius $\varepsilon$ on the boundary of the unit sphere, it was shown in [41] (with a correction as noted in [44]) that a two-term expansion for the average MFPT is given by

$$
\bar{v} \sim \frac{|\Omega|}{4 \varepsilon D}\left[1-\frac{\varepsilon}{\pi} \log \varepsilon+\mathcal{O}(\varepsilon)\right],
$$

where $|\Omega|$ denotes the volume of the unit sphere. This result was derived in [41] by using the Collins method for solving a certain pair of integral equations resulting from a separation of variables approach. A similar result for $\bar{v}$ was obtained in [41] for the 
case of one small elliptical-shaped absorbing window on the boundary of a sphere. For an arbitrary three-dimensional bounded domain with one locally circular absorbing window of radius $\varepsilon$ on its smooth boundary, it was shown in [44] that

$$
\bar{v} \sim \frac{|\Omega|}{4 \varepsilon D}\left[1-\frac{\varepsilon}{\pi} H \log \varepsilon+\mathcal{O}(\varepsilon)\right],
$$

where $H$ denotes the mean curvature of the domain boundary at the center of the absorbing window. In [20] an approximate analytical theory was developed to determine the average MFPT for the case of two circular absorbing windows on the boundary of the unit sphere, with arbitrary window separation. For this two-window case, the average MFPT was determined in terms of an integral and an unspecified $\mathcal{O}(1)$ term, which was estimated from Brownian particle simulations.

The primary goal of this paper is to extend the previous work by calculating a three-term asymptotic expansion for the MFPT for the case of $N$ small locally circular absorbing windows, or traps, on the boundary of the unit sphere. This threeterm asymptotic expansion for the MFPT will show explicitly the significant effects of both the fragmentation of the trap set and the spatial arrangement of the traps on the boundary of the sphere. For the special case where the $N$ traps have a common radius $\varepsilon \ll 1$ and are centered at $x_{j}$ with $\left|x_{j}\right|=1$ for $j=1, \ldots, N$ and $\left|x_{i}-x_{j}\right|=\mathcal{O}(1)$ for $i \neq j$, our results in section 2 show that the average MFPT has the three-term asymptotic expansion

$$
\begin{aligned}
\bar{v}=\frac{|\Omega|}{4 \varepsilon D N}[1 & +\frac{\varepsilon}{\pi} \log \left(\frac{2}{\varepsilon}\right)+\frac{\varepsilon}{\pi}\left(-\frac{9 N}{5}+2(N-2) \log 2+\frac{3}{2}+\frac{4}{N} \mathcal{H}\left(x_{1}, \ldots, x_{N}\right)\right) \\
& \left.+\mathcal{O}\left(\varepsilon^{2} \log \varepsilon\right)\right]
\end{aligned}
$$

where the discrete energy-like function $\mathcal{H}\left(x_{1}, \ldots, x_{N}\right)$ is defined by

$$
\mathcal{H}\left(x_{1}, \ldots, x_{N}\right)=\sum_{i=1}^{N} \sum_{j=i+1}^{N}\left(\frac{1}{\left|x_{i}-x_{j}\right|}-\frac{1}{2} \log \left|x_{i}-x_{j}\right|-\frac{1}{2} \log \left(2+\left|x_{i}-x_{j}\right|\right)\right) .
$$

Results from (1.5) are shown in section 2 to agree very closely with full numerical results computed with the finite element package COMSOL [6]. In section 2 a corresponding three-term result is then given for the case of $N$ arbitrarily shaped, well-separated windows on the boundary of the unit sphere.

The asymptotic analysis in section 2 leading to (1.5), and related results, relies on two essential ingredients. First, it requires detailed properties of the surface Neumann Green's function for the unit sphere and, in particular, the determination of both the subdominant logarithmic singularity and the regular part of this function. This calculation is done in Appendix A. The identification of a weak logarithmic singularity for this Green's function was first made in [23] for the unit sphere and for a general three-dimensional domain in [32], [40], and [44]. Second, the analysis in section 2 requires the introduction of certain logarithmic switchback terms that commonly occur in the asymptotic analysis of certain problems in fluid mechanics (see [27] and [28] for a discussion of logarithmic switchback terms).

In section 3 we use the method of matched asymptotic expansions to derive a three-term asymptotic expansion for the principal eigenvalue $\lambda(\varepsilon)$ of the Laplacian in 
the unit sphere for the case where the boundary of the sphere has $N$ locally circular well-separated traps of small radii. This analysis extends the leading-order asymptotic calculation of [47]. Our results show that, to within the three-term asymptotic approximation, the principal eigenvalue $\lambda(\varepsilon)$ is related to the average MFPT $\bar{v}$ by $\lambda \sim 1 /(D \bar{v})$. Related eigenvalue perturbation and optimization problems for the Laplacian in two-dimensional domains with localized interior traps, or with traps on the domain boundary, are studied in [4], [7], [8], [9], and [24] (see also the references therein).

For the case of $N$ locally circular windows of a common radius, the discrete energy $\mathcal{H}\left(x_{1}, \ldots, x_{N}\right)$ in $(1.5)$ shows explicitly the dependence of the spatial arrangement of the absorbing windows on the MFPT. From (1.5), the average MFPT $\bar{v}$ is minimized, and the corresponding principal eigenvalue of the Laplacian maximized, at the trap configuration $\left\{x_{1}, \ldots, x_{N}\right\}$ that minimizes $\mathcal{H}\left(x_{1}, \ldots, x_{N}\right)$. This discrete variational problem is an extension of the well-known problem of finding the minimum energy configuration of $N$ repelling point charges on the surface of the unit sphere (see [33], [34] and the references therein). In section 4 global optimization methods are used to obtain numerical results for the trap configurations $\left\{x_{1}, \ldots, x_{N}\right\}$ that minimize $\bar{v}$, and the results are compared with corresponding optimal configurations for two classical discrete energy functions: the logarithmic energy and the Coulomb energy. Moreover, a scaling law, with coefficients fitted to the numerical data, is derived to predict the minimum of the discrete energy $\mathcal{H}\left(x_{1}, \ldots, x_{N}\right)$ in the limit $N \rightarrow \infty$. Finally, some open problems are suggested in section 5 .

2. Narrow escape from a spherical domain. In this section we asymptotically calculate the MFPT for escape from the unit sphere when there are $N$ small wellseparated windows on the boundary of the sphere centered at $x_{j}$ with $j=1, \ldots, N$, where $\left|x_{j}\right|=1$. Each window is assumed to have a circular projection onto the tangent plane to the sphere at $x_{j}$ and has a radius of $\varepsilon a_{j}$, where $\varepsilon \ll 1$. The problem for the MFPT $v=v(x)$, written in spherical coordinates, is

$$
\triangle v \equiv v_{r r}+\frac{2}{r} v_{r}+\frac{1}{r^{2} \sin ^{2} \theta} v_{\phi \phi}+\frac{\cot \theta}{r^{2}} v_{\theta}+\frac{1}{r^{2}} v_{\theta \theta}=-\frac{1}{D}, \quad r=|x| \leq 1,
$$

$$
v=0, \quad x \in \partial \Omega_{a}=\bigcup_{j=1}^{N} \partial \Omega_{\varepsilon_{j}}, \quad j=1, \ldots, N ; \quad \partial_{r} v=0, \quad x \in \partial \Omega \backslash \partial \Omega_{a} .
$$

Here each $\partial \Omega_{\varepsilon_{j}}$ for $j=1, \ldots, N$ is a small "circular" cap centered at $\left(\theta_{j}, \phi_{j}\right)$ defined by

$$
\partial \Omega_{\varepsilon_{j}} \equiv\left\{(\theta, \phi) \mid\left(\theta-\theta_{j}\right)^{2}+\sin ^{2}\left(\theta_{j}\right)\left(\phi-\phi_{j}\right)^{2} \leq \varepsilon^{2} a_{j}^{2}\right\} .
$$

The area of $\partial \Omega_{\varepsilon_{j}}$ is $\left|\partial \Omega_{\varepsilon_{j}}\right| \sim \pi \varepsilon^{2} a_{j}^{2}$. In (2.1a), $0 \leq \phi \leq 2 \pi$ is the longitude, $0 \leq \theta \leq \pi$ is the latitude, and the center of the $j$ th window is at $x_{j} \in \partial \Omega$, where $\left|x_{j}\right|=1$ for $j=1, \ldots, N$.

To solve (2.1) asymptotically, we first must calculate the surface Neumann Green's function. For the unit sphere $\Omega$ with volume $|\Omega|=4 \pi / 3$, the surface Neumann Green's function $G_{s}\left(x ; x_{j}\right)$ satisfies

$$
\begin{aligned}
\triangle G_{s} & =\frac{1}{|\Omega|}, \quad x \in \Omega ; \quad \partial_{r} G_{s}=\delta\left(\cos \theta-\cos \theta_{j}\right) \delta\left(\phi-\phi_{j}\right), \quad x \in \partial \Omega ; \\
\int_{\Omega} G_{s} d x & =0 .
\end{aligned}
$$


In terms of spherical coordinates, the points $x \in \partial \Omega, x_{j} \in \partial \Omega$ and the dot product $x \cdot x_{j}$ are given by

$x=(\cos \phi \sin \theta, \sin \phi \sin \theta, \cos \theta), \quad x_{j}=\left(\cos \phi_{j} \sin \theta_{j}, \sin \phi_{j} \sin \theta_{j}, \cos \theta_{j}\right)$,

$\cos \gamma=x \cdot x_{j}$

$\gamma$ denoting the angle between $x$ and $x_{j}$ given by $\cos \gamma=\cos \theta \cos \theta_{j}+\sin \theta \sin \theta_{j} \cos (\phi-$ $\left.\phi_{j}\right)$. The following result for $G_{s}\left(x ; x_{j}\right)$ is derived in Appendix A.

Lemma 2.1. For the unit sphere, the surface Neumann Green's function satisfying (2.2) is given explicitly by

$$
G_{s}\left(x ; x_{j}\right)=\frac{1}{2 \pi\left|x-x_{j}\right|}+\frac{1}{8 \pi}\left(|x|^{2}+1\right)+\frac{1}{4 \pi} \log \left(\frac{2}{1-|x| \cos \gamma+\left|x-x_{j}\right|}\right)-\frac{7}{10 \pi} .
$$

The calculations below for the MFPT require the limiting behavior of $G_{s}$ in (2.4) as $x \rightarrow x_{j} \in \partial \Omega$ when expressed in terms of a local coordinate system $\left(\eta, s_{1}, s_{2}\right)$ whose origin is at the center of the $j$ th absorbing window. We define the local Cartesian coordinate, $y$, together with the local curvilinear coordinates $\eta, s_{1}$, and $s_{2}$, by

$$
y \equiv \varepsilon^{-1}\left(x-x_{j}\right), \quad \eta \equiv \varepsilon^{-1}(1-r), \quad s_{1} \equiv \varepsilon^{-1} \sin \left(\theta_{j}\right)\left(\phi-\phi_{j}\right), \quad s_{2} \equiv \varepsilon^{-1}\left(\theta-\theta_{j}\right) .
$$

From the law of cosines we calculate that

$$
1-|x| \cos \gamma=\frac{1}{2}\left[\left|x-x_{j}\right|^{2}-\left(|x|^{2}-1\right)\right] \sim \frac{1}{2}\left[\mathcal{O}\left(\varepsilon^{2}\right)-\left((1-\varepsilon \eta)^{2}-1\right)\right] \sim \varepsilon \eta+\mathcal{O}\left(\varepsilon^{2}\right) .
$$

Therefore, upon substituting (2.6) and (2.5) into (2.4), we obtain as $x \rightarrow x_{j}$ that

$$
G_{s}\left(x ; x_{j}\right)=\frac{1}{2 \pi \varepsilon|y|}-\frac{1}{4 \pi} \log \left(\frac{\varepsilon}{2}\right)-\frac{1}{4 \pi} \log (|y|+\eta)-\frac{9}{20 \pi}+\mathcal{O}(\varepsilon) .
$$

The weak logarithmic singularity in (2.7) on $\eta=0$ was observed previously for the sphere in [23] (see page 247 of [23]) and for general domains in [40], [32], and [44]. Our calculation in Appendix A has identified the regular part of the singularity structure for $G_{s}$ in (2.7), which is needed below to obtain a three-term expansion for the MFPT.

By retaining linear and quadratic terms for the mapping $x-x_{j} \mapsto\left(\eta, s_{1}, s_{2}\right)$, a lengthy but straightforward calculation, which we omit, shows that for $x \rightarrow x_{j}$

$$
\frac{1}{|y|}=\frac{1}{\rho}+\frac{\varepsilon}{2 \rho^{3}}\left[\eta\left(s_{1}^{2}+s_{2}^{2}\right)-s_{1}^{2} s_{2} \cot \theta_{j}\right]+\mathcal{O}\left(\varepsilon^{2}\right), \quad \rho \equiv\left(\eta^{2}+s_{1}^{2}+s_{2}^{2}\right)^{1 / 2} .
$$

In order to obtain the local representation of the surface Neumann Green's function with an error of $\mathcal{O}(\varepsilon)$, as required for the asymptotic analysis below, we substitute (2.8) into (2.7) to obtain for $x \rightarrow x_{j}$ that

$$
\begin{aligned}
G_{s}\left(x ; x_{j}\right)= & \frac{1}{2 \pi \varepsilon \rho}-\frac{1}{4 \pi} \log \left(\frac{\varepsilon}{2}\right)+\frac{1}{4 \pi}\left[\frac{\eta\left(s_{1}^{2}+s_{2}^{2}\right)}{\rho^{3}}-\frac{s_{1}^{2} s_{2} \cot \theta_{j}}{\rho^{3}}\right] \\
& -\frac{1}{4 \pi} \log (\rho+\eta)-\frac{9}{20 \pi}+\mathcal{O}(\varepsilon) .
\end{aligned}
$$

We now solve (2.1) in the limit $\varepsilon \rightarrow 0$ by using the method of matched asymptotic expansions. In the outer region away from the absorbing windows we expand the outer 
solution as

$$
v \sim \varepsilon^{-1} v_{0}+v_{1}+\varepsilon \log \left(\frac{\varepsilon}{2}\right) v_{2}+\varepsilon v_{3}+\cdots .
$$

Here $v_{0}$ is an unknown constant, while $v_{1}, v_{2}$, and $v_{3}$ are functions to be determined. As shown below, the third nonanalytic term in $\varepsilon$ in (2.10) arises as a result of the term in (2.9) with logarithmic dependence on $\varepsilon$. In addition, we show below that one must add a further term of the form $\log (\varepsilon / 2) \chi_{0}$ directly between the first and second terms in $(2.10)$, where $\chi_{0}$ is a certain constant. Such terms are called switchback terms in singular perturbation theory, and they have a long history in the study of certain ODE and PDE models in fluid mechanics (cf. [27], [28]).

We first substitute $(2.10)$ into $(2.1)$ to obtain that $v_{k}$, for $k=1, \ldots, 3$, satisfies

$$
\triangle v_{k}=-\frac{1}{D} \delta_{k 1}, \quad x \in \Omega ; \quad \partial_{n} v_{k}=0, \quad x \in \partial \Omega \backslash\left\{x_{1}, \ldots, x_{N}\right\},
$$

where $\delta_{k 1}=1$ if $k=1$ and $\delta_{k 1}=0$ for $k>1$. The analysis below yields appropriate singularity behaviors for each $v_{k}$ as $x \rightarrow x_{j}$ for $j=1, \ldots, N$. In the inner region near the $j$ th absorbing window we introduce the local coordinates $\left(\eta, s_{1}, s_{2}\right)$ as defined in (2.5), and we pose the inner expansion

$$
v \sim \varepsilon^{-1} w_{0}+\log \left(\frac{\varepsilon}{2}\right) w_{1}+w_{2}+\cdots .
$$

We substitute (2.12) into (2.1) after first transforming (2.1a) in terms of the local coordinate system (2.5) as outlined in Appendix B. In the limit $\varepsilon \rightarrow 0$, this yields a sequence of problems for $w_{k}$ for $k=0,1,2$ given by

$$
\partial_{\eta} w_{k}=0 \quad \text { on } \quad \eta=0, s_{1}^{2}+s_{2}^{2} \geq a_{j}^{2} ; \quad w_{k}=0 \quad \text { on } \quad \eta=0, s_{1}^{2}+s_{2}^{2} \leq a_{j}^{2},
$$

where $\delta_{22}=1$ and $\delta_{k 2}=0$ if $k=0,1$. In (2.13a), $\mathcal{F}_{2}$ is defined by (2.13c)

$$
\mathcal{F}_{2} \equiv 2\left(\eta w_{0 \eta \eta}+w_{0 \eta}\right)-\cot \theta_{j}\left(w_{0 s_{2}}-2 s_{2} w_{0 s_{1} s_{1}}\right), \quad \eta \geq 0, \quad-\infty<s_{1}, s_{2}<\infty .
$$

The leading-order matching condition is that $w_{0} \sim v_{0}$ as $\rho \equiv\left(\eta^{2}+s_{1}^{2}+s_{2}^{2}\right)^{1 / 2} \rightarrow \infty$. Therefore, we write

$$
w_{0}=v_{0}\left(1-w_{c}\right),
$$

where $v_{0}$ is a constant to be determined, and $w_{c}$ is the solution satisfying $w_{c} \rightarrow 0$ as $\rho \rightarrow \infty$ to

$$
\partial_{\eta} w_{c}=0 \quad \text { on } \quad \eta=0, s_{1}^{2}+s_{2}^{2} \geq a_{j}^{2} ; \quad w_{c}=1 \quad \text { on } \quad \eta=0, s_{1}^{2}+s_{2}^{2} \leq a_{j}^{2} .
$$

This is the well-known electrified disk problem in electrostatics (cf. [22]), whose solution is (see page 38 of [12])

$$
w_{c}=\frac{2}{\pi} \int_{0}^{\infty} \frac{\sin \mu}{\mu} e^{-\mu \eta / a_{j}} J_{0}\left(\frac{\mu \sigma}{a_{j}}\right) d \mu=\frac{2}{\pi} \sin ^{-1}\left(\frac{a_{j}}{L}\right), \quad \sigma \equiv\left(s_{1}^{2}+s_{2}^{2}\right)^{1 / 2},
$$


where $J_{0}(z)$ is the Bessel function of the first kind of order zero, and $L=L(\eta, \sigma)$ is defined by

$$
L(\eta, \sigma) \equiv \frac{1}{2}\left(\left[\left(\sigma+a_{j}\right)^{2}+\eta^{2}\right]^{1 / 2}+\left[\left(\sigma-a_{j}\right)^{2}+\eta^{2}\right]^{1 / 2}\right) .
$$

From either an asymptotic expansion of the integral representation of $w_{c}$ using Laplace's method or, alternatively, from a direct calculation of the simple exact solution for $w_{c}$ given in (2.16a), we readily obtain the far-field behavior

$$
w_{c} \sim \frac{2 a_{j}}{\pi}\left(\frac{1}{\rho}+\frac{a_{j}^{2}}{6}\left(\frac{1}{\rho^{3}}-\frac{3 \eta^{2}}{\rho^{5}}\right)+\cdots\right) \quad \text { as } \quad \rho \rightarrow \infty,
$$

which is uniformly valid in $\eta, s_{1}$, and $s_{2}$. Therefore, from (2.14) and (2.17), the far-field expansion for $w_{0}$ is

$$
w_{0} \sim v_{0}\left(1-\frac{c_{j}}{\rho}+\mathcal{O}\left(\rho^{-3}\right)\right) \quad \text { as } \quad \rho \rightarrow \infty, \quad c_{j} \equiv \frac{2 a_{j}}{\pi},
$$

where $c_{j}$ is the electrostatic capacitance of the circular disk of radius $a_{j}$. Next, we write the matching condition that the near-field behavior of the outer expansion (2.10) must agree with the far-field behavior of the inner expansion (2.12), so that

$$
\frac{v_{0}}{\varepsilon}+v_{1}+\varepsilon \log \left(\frac{\varepsilon}{2}\right) v_{2}+\varepsilon v_{3}+\cdots \sim \frac{v_{0}}{\varepsilon}\left(1-\frac{c_{j}}{\rho}+\cdots\right)+\log \left(\frac{\varepsilon}{2}\right) w_{1}+w_{2}+\cdots
$$

Therefore, since $\rho \sim \varepsilon^{-1}\left|x-x_{j}\right|$, we obtain that $v_{1}$ must satisfy (2.11) with the singular behavior $v_{1} \sim-v_{0} c_{j} /\left|x-x_{j}\right|$ as $x \rightarrow x_{j}$ for $j=1, \ldots, N$. This problem for $v_{1}$ can be written in distributional form as

$$
\triangle v_{1}=-\frac{1}{D}, \quad x \in \Omega ;\left.\quad \partial_{r} v_{1}\right|_{r=1}=-2 \pi v_{0} \sum_{j=1}^{N} \frac{c_{j}}{\sin \theta_{j}} \delta\left(\theta-\theta_{j}\right) \delta\left(\phi-\phi_{j}\right) .
$$

By applying the divergence theorem, (2.20) has a solution only when $v_{0}$ is given by

$$
v_{0}=\frac{|\Omega|}{2 \pi D N \bar{c}}, \quad \bar{c} \equiv \frac{1}{N} \sum_{j=1}^{N} c_{j}, \quad c_{j}=\frac{2 a_{j}}{\pi} .
$$

Thus, the solvability condition for the problem for $v_{1}$ determines the unknown leadingorder constant term $v_{0}$ in the outer expansion. The solution to $(2.20)$ is then written as a superposition over the surface Neumann Green's function $G_{s}\left(x ; x_{j}\right)$, with $\int_{\Omega} G_{s}\left(x ; x_{j}\right) d x=0$, together with an unknown constant $\chi$, as

$$
v_{1}=-2 \pi v_{0} \sum_{i=1}^{N} c_{i} G_{s}\left(x ; x_{i}\right)+\chi, \quad \chi \equiv|\Omega|^{-1} \int_{\Omega} v_{1} d x .
$$

Next, we expand $v_{1}$ as $x \rightarrow x_{j}$ by using the near-field expansion of the surface Neumann Green's function given in (2.9). Upon substituting the resulting expression into the matching condition (2.19) we obtain

$$
\begin{aligned}
& \frac{v_{0}}{\varepsilon}\left(1-\frac{c_{j}}{\rho}\right)+\frac{v_{0} c_{j}}{2} \log \left(\frac{\varepsilon}{2}\right)+\chi+\frac{v_{0} c_{j}}{2}\left[\log (\eta+\rho)-\frac{\eta\left(s_{1}^{2}+s_{2}^{2}\right)}{\rho^{3}}+\frac{s_{1}^{2} s_{2} \cot \theta_{j}}{\rho^{3}}\right]+B_{j} \\
& \quad+\varepsilon \log \left(\frac{\varepsilon}{2}\right) v_{2}+\varepsilon v_{3}+\cdots \sim \frac{v_{0}}{\varepsilon}\left(1-\frac{c_{j}}{\rho}+\mathcal{O}\left(\rho^{-3}\right)\right)+\log \left(\frac{\varepsilon}{2}\right) w_{1}+w_{2}+\cdots .
\end{aligned}
$$

Copyright (c) by SIAM. Unauthorized reproduction of this article is prohibited. 
Here the constant $B_{j}$ is defined by

$$
B_{j}=-2 \pi v_{0}\left(-\frac{9}{20 \pi} c_{j}+\sum_{\substack{i=1 \\ i \neq j}}^{N} c_{i} G_{s j i}\right), \quad G_{s j i} \equiv G_{s}\left(x_{j} ; x_{i}\right) .
$$

We compare the $\mathcal{O}(\log \varepsilon)$ terms on both sides of (2.23), which suggests that $w_{1} \sim$ $v_{0} c_{j} / 2$ as $\rho \rightarrow \infty$. However, this leads to a problem for $v_{2}$ with no solution. In order to obtain a solvable equation for $v_{2}$, we must write $\chi$ in the form

$$
\chi=\log \left(\frac{\varepsilon}{2}\right) \chi_{0}+\chi_{1},
$$

where $\chi_{0}$ and $\chi_{1}$ are constants, independent of $\varepsilon$, to be found. This choice for $\chi$ is equivalent to inserting a constant term of order $\mathcal{O}(\log \varepsilon)$ between $v_{0}$ and $v_{1}$ in the outer expansion (2.10). With this choice of $\chi$ in (2.23), the matching condition (2.23) enforces that $w_{1} \sim \chi_{0}+v_{0} c_{j} / 2$ as $\rho \rightarrow \infty$. The solution $w_{1}$ to (2.13) that satisfies this far-field behavior is

$$
w_{1}=\left(\frac{v_{0} c_{j}}{2}+\chi_{0}\right)\left(1-w_{c}\right),
$$

where $w_{c}$, given explicitly in (2.16), is the solution to (2.15). Therefore, using (2.17), we obtain the far-field behavior

$$
w_{1} \sim\left(\frac{v_{0} c_{j}}{2}+\chi_{0}\right)\left(1-\frac{c_{j}}{\rho}+\mathcal{O}\left(\rho^{-3}\right)\right) .
$$

Next, we substitute (2.27) into the matching condition (2.23) and use $\rho \sim \varepsilon^{-1} \mid x-$ $x_{0} \mid$. This yields that the solution $v_{2}$ to $(2.11)$ has the singular behavior $v_{2} \sim$ $-\left(\frac{v_{0} c_{j}}{2}+\chi_{0}\right) c_{j} /\left|x-x_{j}\right|$ as $x \rightarrow x_{j}$. Therefore, $v_{2}$ satisfies

$$
\triangle v_{2}=0, \quad x \in \Omega ;\left.\quad \partial_{r} v_{2}\right|_{r=1}=-2 \pi \sum_{j=1}^{N} c_{j}\left(\frac{v_{0} c_{j}}{2}+\chi_{0}\right) \frac{\delta\left(\theta-\theta_{j}\right) \delta\left(\phi-\phi_{j}\right)}{\sin \theta_{j}} .
$$

By using the divergence theorem, we obtain that (2.28) is solvable only when $\chi_{0}$ is given by

$$
\chi_{0}=-\frac{v_{0}}{2 N \bar{c}} \sum_{j=1}^{N} c_{j}^{2} .
$$

Then, the solution for $v_{2}$ can be written in terms of the surface Neumann Green's function as

$$
v_{2}=-2 \pi \sum_{i=1}^{N} c_{i}\left(\frac{v_{0} c_{i}}{2}+\chi_{0}\right) G_{s}\left(x ; x_{i}\right)+\chi_{2} .
$$

Next, we match the $\mathcal{O}(1)$ terms in (2.23) with $\chi$ as given in (2.25). We obtain that $w_{2}$ satisfies (2.13) with the far-field behavior

$w_{2} \sim B_{j}+\chi_{1}+\frac{v_{0} c_{j}}{2}\left[\log (\eta+\rho)-\frac{\eta\left(s_{1}^{2}+s_{2}^{2}\right)}{\rho^{3}}\right]+\left(\frac{v_{0} c_{j}}{2 \rho^{3}}\right) s_{1}^{2} s_{2} \cot \theta_{j} \quad$ as $\quad \rho \rightarrow \infty$. 
By superposition, we decompose the solution to this problem for $w_{2}$ in the form

$$
w_{2}=\left(B_{j}+\chi_{1}\right)\left(1-w_{c}\right)+v_{0} w_{2 e}+v_{0} w_{2 o},
$$

where $w_{c}$ is the solution to the electrified disk problem (2.15). Upon writing $w_{0}=$ $v_{0}\left(1-w_{c}\right)$ to calculate $\mathcal{F}_{2}$ in $(2.13 \mathrm{c})$, we set $w_{2 e}$ to be the solution to

$$
w_{2 e \eta \eta}+w_{2 e s_{1} s_{1}}+w_{2 e s_{2} s_{2}}=-2 w_{c \eta}-2 \eta w_{c \eta \eta}, \quad \eta \geq 0, \quad-\infty<s_{1}, s_{2}<\infty,
$$

$$
\partial_{\eta} w_{2 e}=0 \quad \text { on } \quad \eta=0, s_{1}^{2}+s_{2}^{2} \geq a_{j}^{2} ; \quad w_{2 e}=0 \quad \text { on } \quad \eta=0, s_{1}^{2}+s_{2}^{2} \leq a_{j}^{2},
$$

$$
w_{2 e} \sim \frac{c_{j}}{2} \log (\eta+\rho)-\frac{c_{j}}{2 \rho^{3}} \eta\left(s_{1}^{2}+s_{2}^{2}\right) \quad \text { as } \quad \rho \rightarrow \infty .
$$

Moreover, $w_{2 o}$ is taken to be the solution of

$w_{2 o \eta \eta}+w_{2 o s_{1} s_{1}}+w_{2 o s_{2} s_{2}}=\cot \theta_{j}\left(w_{c s_{2}}-2 s_{2} w_{c s_{1} s_{1}}\right), \quad \eta \geq 0, \quad-\infty<s_{1}, s_{2}<\infty$,

$$
\partial_{\eta} w_{2 o}=0 \quad \text { on } \quad \eta=0, s_{1}^{2}+s_{2}^{2} \geq a_{j}^{2} ; \quad w_{2 o}=0 \quad \text { on } \quad \eta=0, s_{1}^{2}+s_{2}^{2} \leq a_{j}^{2},
$$

$$
w_{2 o} \sim \frac{c_{j}}{2 \rho^{3}} s_{1}^{2} s_{2} \cot \theta_{j} \quad \text { as } \quad \rho \rightarrow \infty .
$$

In Appendix B we show that the inhomogeneous terms given by the right-hand sides of (2.33a) and (2.34a) lead explicitly to the leading-order far-field asymptotic behavior as written in $(2.33 \mathrm{c})$ and $(2.34 \mathrm{c})$.

The solution $v_{1}$ in (2.22) involves an as yet unknown constant $\chi_{1}$ from (2.25). In the determination of $\chi_{1}$ below from a solvability condition applied to the problem for $v_{3}$, we must have identified all of the monopole terms of the form $b / \rho$ as $\rho \rightarrow \infty$ for some constant $b$ arising from the far-field behavior of each term in the decomposition (2.32) of $w_{2}$. It is only these monopole terms that give nonvanishing contributions in the solvability condition determining $\chi_{1}$. Clearly, the first term $\left(B_{j}+\chi_{1}\right)\left(1-w_{c}\right)$ in (2.32) yields a monopole term from (2.17). However, upon solving the problem for $w_{2 e}$ exactly as in Lemma B.1 of Appendix B, we obtain that $w_{2 e}$ also yields a monopole term, and it has the far-field behavior

$$
w_{2 e}=\frac{c_{j}}{2} \log (\eta+\rho)-\frac{c_{j}}{2 \rho^{3}} \eta\left(s_{1}^{2}+s_{2}^{2}\right)-\frac{c_{j} \kappa_{j}}{\rho}+\mathcal{O}\left(\rho^{-2}\right) \quad \text { as } \quad \rho \rightarrow \infty,
$$

where $\kappa_{j}$ is given explicitly by

$$
\kappa_{j}=\frac{c_{j}}{2}\left[2 \log 2-\frac{3}{2}+\log a_{j}\right] .
$$

Alternatively, the solution $w_{2 o}$ to (2.34) is odd in $s_{2}$ and, hence, does not generate a monopole term at infinity. An explicit analytical solution for $w_{2 o}$ is given in Lemma B.2 of Appendix B.

In this way, we obtain that the solution $w_{2}$ to $(2.13)$ with leading-order far-field behavior (2.31) generates further terms in the far-field behavior of the form

$$
\begin{array}{r}
w_{2} \sim\left(B_{j}+\chi_{1}\right)\left(1-\frac{c_{j}}{\rho}\right)+\frac{v_{0} c_{j}}{2}\left[\log (\eta+\rho)-\frac{\eta}{\rho^{3}}\left(s_{1}^{2}+s_{2}^{2}\right)+\frac{s_{1}^{2} s_{2}}{\rho^{3}} \cot \theta_{j}\right. \\
\left.-\frac{2 \kappa_{j}}{\rho}+\mathcal{O}\left(\rho^{-2}\right)\right] \quad \text { as } \quad \rho \rightarrow \infty .
\end{array}
$$

Copyright $@$ by SIAM. Unauthorized reproduction of this article is prohibited. 
Finally, we substitute (2.37) into the matching condition (2.23). The two monopole terms in (2.37) determine the singular behavior for the solution $v_{3}$ of $(2.11)$ as

$$
v_{3} \sim-\frac{c_{j}\left(B_{j}+\chi_{1}+v_{0} \kappa_{j}\right)}{\left|x-x_{j}\right|} \quad \text { as } \quad x \rightarrow x_{j} .
$$

In distributional form, this problem for $v_{3}$ is equivalent to

$$
\triangle v_{3}=0, \quad x \in \Omega ;\left.\quad \partial_{r} v_{3}\right|_{r=1}=-2 \pi \sum_{j=1}^{N} c_{j}\left(B_{j}+\chi_{1}+v_{0} \kappa_{j}\right) \frac{\delta\left(\theta-\theta_{j}\right) \delta\left(\phi-\phi_{j}\right)}{\sin \theta_{j}} .
$$

The solvability condition for (2.39), obtained by using the divergence theorem, determines $\chi_{1}$ as

$$
\chi_{1}=-\frac{1}{N \bar{c}} \sum_{j=1}^{N} c_{j}\left[B_{j}+v_{0} \kappa_{j}\right]
$$

Then, upon using (2.24) for $B_{j}$, we can write $\chi_{1}$ as the sum of two terms, one of which involves a quadratic form in terms of the capacitance vector $\mathcal{C}^{T} \equiv\left(c_{1}, \ldots, c_{N}\right)$, as

$$
\chi_{1}=\frac{2 \pi v_{0}}{N \bar{c}} p_{c}\left(x_{1}, \ldots, x_{N}\right)-\frac{v_{0}}{N \bar{c}} \sum_{j=1}^{N} c_{j} \kappa_{j}, \quad p_{c}\left(x_{1}, \ldots, x_{N}\right) \equiv \mathcal{C}^{T} \mathcal{G}_{s} \mathcal{C}
$$

Here $\kappa_{j}$ is given in (2.36) and $\mathcal{G}_{s}$ is the Green's function matrix defined in terms of $G_{s}\left(x_{i} ; x_{j}\right)$ by

$$
\mathcal{G}_{s} \equiv\left(\begin{array}{cccc}
R & G_{s 12} & \cdots & G_{s 1 N} \\
G_{s 21} & R & \cdots & G_{s 2 N} \\
\vdots & \vdots & \ddots & \vdots \\
G_{s N 1} & \cdots & G_{s N, N-1} & R
\end{array}\right), \quad R=-\frac{9}{20 \pi}, \quad G_{s i j} \equiv G_{s}\left(x_{i} ; x_{j}\right)
$$

Finally, we substitute $(2.21)$ for $v_{0}$ together with (2.22) for $v_{1}$, with $\chi$ as determined by $(2.25),(2.29)$, and $(2.41)$, into the outer expansion (2.10). This leads to the following main result.

Principal ReSult 2.2. For $\varepsilon \rightarrow 0$, the asymptotic solution to (2.1) is given in the outer region $\left|x-x_{j}\right| \gg \mathcal{O}(\varepsilon)$ for $j=1, \ldots, N$ by

$$
\begin{aligned}
v=\frac{|\Omega|}{2 \pi \varepsilon D N \bar{c}}[1 & +\varepsilon \log \left(\frac{2}{\varepsilon}\right) \frac{\sum_{j=1}^{N} c_{j}^{2}}{2 N \bar{c}}-2 \pi \varepsilon \sum_{j=1}^{N} c_{j} G_{s}\left(x ; x_{j}\right) \\
& \left.+\frac{2 \pi \varepsilon}{N \bar{c}} p_{c}\left(x_{1}, \ldots, x_{N}\right)-\frac{\varepsilon}{N \bar{c}} \sum_{j=1}^{N} c_{j} \kappa_{j}+\mathcal{O}\left(\varepsilon^{2} \log \varepsilon\right)\right] .
\end{aligned}
$$

Here $c_{j}=2 a_{j} / \pi$ is the capacitance of the jth circular absorbing window of radius $\varepsilon a_{j}$, $\bar{c} \equiv N^{-1}\left(c_{1}+\cdots+c_{N}\right),|\Omega|=4 \pi / 3, \kappa_{j}$ is defined in $(2.36), G_{s}\left(x ; x_{j}\right)$ is the surface Neumann Green's function given in $(2.4)$, and $p_{c}\left(x_{1}, \ldots, x_{N}\right)$ is the quadratic form defined in (2.41). Since $\int_{\Omega} G_{s} d x=0$, then $\bar{v}=|\Omega|^{-1} \int_{\Omega} v d x$ is given by

Copyright (c) by SIAM. Unauthorized reproduction of this article is prohibited. 


$$
\begin{gathered}
\bar{v}=\frac{|\Omega|}{2 \pi \varepsilon D N \bar{c}}\left[1+\varepsilon \log \left(\frac{2}{\varepsilon}\right) \frac{\sum_{j=1}^{N} c_{j}^{2}}{2 N \bar{c}}+\frac{2 \pi \varepsilon}{N \bar{c}} p_{c}\left(x_{1}, \ldots, x_{N}\right)\right. \\
\left.-\frac{\varepsilon}{N \bar{c}} \sum_{j=1}^{N} c_{j} \kappa_{j}+\mathcal{O}\left(\varepsilon^{2} \log \varepsilon\right)\right] .
\end{gathered}
$$

For the case of one circular window of radius $\varepsilon a$, we set $N=1, c_{1}=2 a / \pi$, and $a_{1}=a$ in (2.43), (2.36), and (2.44) to get

$$
\begin{aligned}
\bar{v} & =\frac{|\Omega|}{4 \varepsilon a D}\left[1+\frac{\varepsilon a}{\pi} \log \left(\frac{2}{\varepsilon a}\right)+\frac{\varepsilon a}{\pi}\left(-\frac{9}{5}-2 \log 2+\frac{3}{2}\right)+\mathcal{O}\left(\varepsilon^{2} \log \varepsilon\right)\right], \\
v(x) & =\bar{v}-\frac{|\Omega|}{D} G_{s}\left(x ; x_{1}\right) .
\end{aligned}
$$

For an initial position at the origin, i.e., $x=(0,0)$, then with $G_{s}\left(0 ; x_{1}\right)=-3 /(40 \pi)$ from (2.4), (2.45) becomes

$$
v(0)=\frac{|\Omega|}{4 \varepsilon a D}\left[1+\frac{\varepsilon a}{\pi} \log \left(\frac{2}{\varepsilon a}\right)-\frac{2 \varepsilon a \log 2}{\pi}+\mathcal{O}\left(\varepsilon^{2} \log \varepsilon\right)\right] .
$$

For the case of one circular absorbing window of radius $\varepsilon$ (i.e., $a=1$ ), it was derived in [41] that

$$
\bar{v} \sim \frac{|\Omega|}{4 \varepsilon D}\left[1+\frac{\varepsilon}{\pi} \log \left(\frac{1}{\varepsilon}\right)+\mathcal{O}(\varepsilon)\right] .
$$

The original result in equation (3.52) of [41] omits the $\pi$ term in (2.47) due to an omission of an extra factor of $\pi$ on the left-hand side of the equation above (3.52) of [41]. This was corrected in [44]. Our result (2.45) agrees asymptotically with that of (2.47) and determines the $\mathcal{O}(\varepsilon)$ term to $\bar{v}$ explicitly. More importantly, our main result in Principal Result 2.2 generalizes that of [41] to the case of $N$ circular absorbing windows of different radii on the unit sphere and provides the $\mathcal{O}(\varepsilon)$ term that accounts for the specific locations of the traps on the unit sphere.

A further interesting special case of Principal Result 2.2 is when there are $N$ circular absorbing windows of a common radius $\varepsilon$. Then, upon setting $c_{j}=2 / \pi$, together with $a_{j}=1$ for $j=1, \ldots, N$ in (2.36), (2.44) reduces to

$$
\begin{aligned}
\bar{v}=\frac{|\Omega|}{4 \varepsilon D N}[1 & +\frac{\varepsilon}{\pi} \log \left(\frac{2}{\varepsilon}\right)+\frac{\varepsilon}{\pi}\left(-\frac{9}{5}+\frac{8 \pi}{N} \sum_{i=1}^{N} \sum_{j=i+1}^{N} G_{s}\left(x_{i} ; x_{j}\right)-2 \log 2+\frac{3}{2}\right) \\
& \left.+\mathcal{O}\left(\varepsilon^{2} \log \varepsilon\right)\right] .
\end{aligned}
$$

From $(2.4)$, we readily calculate the interaction term $G_{s}\left(x_{i} ; x_{j}\right)$ in $(2.48)$ as

$$
\begin{aligned}
G_{s}\left(x_{i} ; x_{j}\right) & =-\frac{9}{20 \pi}+\frac{1}{2 \pi}\left(\frac{1}{\left|x_{i}-x_{j}\right|}-\frac{1}{2} \log \left[\sin ^{2}\left(\frac{\gamma_{i j}}{2}\right)+\sin \left(\frac{\gamma_{i j}}{2}\right)\right]\right), \\
\cos \left(\gamma_{i j}\right) & =x_{i} \cdot x_{j}
\end{aligned}
$$

where $\gamma_{i j}$ denotes the angle between $x_{i}$ and $x_{j}$. Therefore, (2.48) becomes 


$$
\begin{aligned}
\bar{v}=\frac{|\Omega|}{4 \varepsilon D N}[1 & +\frac{\varepsilon}{\pi} \log \left(\frac{2}{\varepsilon}\right)+\frac{\varepsilon}{\pi}\left(-\frac{9 N}{5}-2 \log 2+\frac{3}{2}+\frac{4}{N} \tilde{\mathcal{H}}\left(x_{1}, \ldots, x_{N}\right)\right) \\
& \left.+\mathcal{O}\left(\varepsilon^{2} \log \varepsilon\right)\right],
\end{aligned}
$$

where the discrete sum $\tilde{\mathcal{H}}\left(x_{1}, \ldots, x_{N}\right)$ with $\left|x_{j}\right|=1$ and $\cos \gamma_{i j}=x_{i} \cdot x_{j}$ for $i, j=$ $1, \ldots, N$ is defined by

$$
\tilde{\mathcal{H}}\left(x_{1}, \ldots, x_{N}\right)=\sum_{i=1}^{N} \sum_{j>i}^{N}\left(\frac{1}{\left|x_{i}-x_{j}\right|}-\frac{1}{2} \log \left[\sin ^{2}\left(\frac{\gamma_{i j}}{2}\right)+\sin \left(\frac{\gamma_{i j}}{2}\right)\right]\right) .
$$

Equivalently, we can write $\bar{v}$ in the alternative form

$$
\begin{aligned}
\bar{v}=\frac{|\Omega|}{4 \varepsilon D N}[1 & +\frac{\varepsilon}{\pi} \log \left(\frac{2}{\varepsilon}\right)+\frac{\varepsilon}{\pi}\left(-\frac{9 N}{5}+2(N-2) \log 2+\frac{3}{2}+\frac{4}{N} \mathcal{H}\left(x_{1}, \ldots, x_{N}\right)\right) \\
& \left.+\mathcal{O}\left(\varepsilon^{2} \log \varepsilon\right)\right]
\end{aligned}
$$

where $\mathcal{H}\left(x_{1}, \ldots, x_{N}\right)$ is defined by

$$
\mathcal{H}\left(x_{1}, \ldots, x_{N}\right)=\sum_{i=1}^{N} \sum_{j=i+1}^{N}\left(\frac{1}{\left|x_{i}-x_{j}\right|}-\frac{1}{2} \log \left|x_{i}-x_{j}\right|-\frac{1}{2} \log \left(2+\left|x_{i}-x_{j}\right|\right)\right) .
$$

The first term in $\mathcal{H}$ is the usual Coulomb singularity in three dimensions, whereas the second term in $(2.51 \mathrm{~b})$ represents a contribution from surface diffusion on the boundary of the sphere, similar to that studied in [7].

As a remark, for the case of $N$ circular absorbing windows of a common radius $\varepsilon$, the average MFPT, $\bar{v}$, is minimized in the limit $\varepsilon \rightarrow 0$ at the trap configuration $\left\{x_{1}, \ldots, x_{N}\right\}$ that minimizes the discrete sum $\mathcal{H}\left(x_{1}, \ldots, x_{N}\right)$ on the unit sphere $\left|x_{j}\right|=1$ for $j=1, \ldots, N$. The classic discrete variational problem of minimizing either the Coulomb energy $\sum_{i=1}^{N} \sum_{j=i+1}^{N}\left|x_{i}-x_{j}\right|^{-1}$ or the logarithmic energy $-\sum_{i=1}^{N} \sum_{j=i+1}^{N} \log \left|x_{i}-x_{j}\right|$ on the unit sphere has a long history in approximation theory (see [11], [15], [3], [33], [34], [25], [17] and the references therein).

Next, we validate our asymptotic result (2.51) with full numerical results. In Figure 2.1 we compare our asymptotic results for the average MFPT $\bar{v}$ versus $\varepsilon$ with those computed from full numerical simulations using the COMSOL finite element package [6]. The comparisons are done for $N=1, N=2$, and $N=4$, identical traps equally spaced on the surface of the unit sphere (see the caption of Figure 2.1). Table 2.1 compares the two-term and three-term predictions for $\bar{v}$ from (2.51) with corresponding full numerical results computed using COMSOL. Note that the threeterm expansion for $\bar{v}$ in (2.51) agrees well with full numerical results even when $\varepsilon=0.5$. For $\varepsilon=0.5$ and $N=4$, we calculate $N \pi \varepsilon^{2} /(4 \pi) \approx 0.20$, so that the absorbing windows occupy roughly $25 \%$ of the surface area of the unit sphere. For this challenging test of perturbation theory, the last row and last three columns in Table 2.1 show that the three-term asymptotic result for the average MFPT differs from the full numerical result by only about $10 \%$.

Finally, we remark that our main result (2.44) can also readily be used for absorbing windows that are not circular. To treat this modification of (2.1), the following 


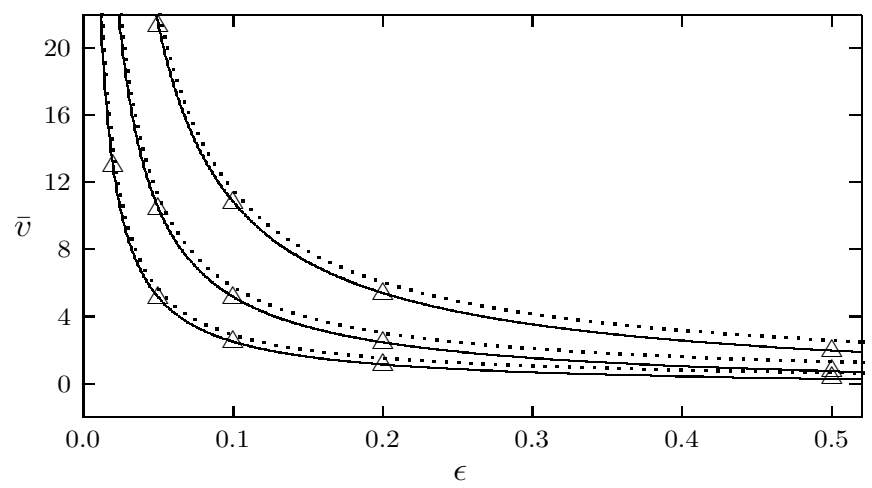

Fig. 2.1. Plot of the average MFPT $\bar{v}$ versus $\varepsilon$ from (2.51) with $D=1$ for either one, two, or four identical windows on the surface of the unit sphere. The solid curves are the three-term expansion from (2.51), while the dotted curves are the truncation of (2.51) to two terms. The triangles denote the full numerical results computed from COMSOL [6]. Top curves: $N=1$. Middle curves: $N=2$ with antipodal windows. Bottom curves: $N=4$ with windows at the north and south poles and two windows equally spaced on the equator.

TABLE 2.1

Comparison of asymptotic and full numerical results for $\bar{v}$ for either $N=1, N=2$, or $N=4$, identical circular windows of radius $\varepsilon$ equidistantly placed on the surface of the unit sphere (see the caption of Figure 2.1). Here $\bar{v}_{2}$ is the two-term asymptotic result obtained by omitting the $\mathcal{O}(\varepsilon)$ term in (2.51), $\bar{v}_{3}$ is the three-term asymptotic result of (2.51), and $\bar{v}_{n}$ is the full numerical result computed from COMSOL [6].

\begin{tabular}{c|ccc|ccc|ccc}
\hline & \multicolumn{3}{|c}{$N=1$} & \multicolumn{3}{c}{$N=2$} \\
\hline$\varepsilon$ & $\bar{v}_{2}$ & $\bar{v}_{3}$ & $\bar{v}_{n}$ & $\bar{v}_{2}$ & $\bar{v}_{3}$ & $\bar{v}_{n}$ & $\bar{v}_{2}$ & $\bar{v}_{3}$ & $\bar{v}_{n}$ \\
\hline 0.02 & 53.89 & 53.33 & 52.81 & 26.95 & 26.42 & 26.12 & 13.47 & 13.11 & 12.99 \\
0.05 & 22.17 & 21.61 & 21.35 & 11.09 & 10.56 & 10.43 & 5.54 & 5.18 & 5.12 \\
0.10 & 11.47 & 10.91 & 10.78 & 5.74 & 5.21 & 5.14 & 2.87 & 2.51 & 2.47 \\
0.20 & 6.00 & 5.44 & 5.36 & 3.00 & 2.47 & 2.44 & 1.50 & 1.14 & 1.13 \\
0.50 & 2.56 & 1.99 & 1.96 & 1.28 & 0.75 & 0.70 & 0.64 & 0.28 & 0.30 \\
\hline
\end{tabular}

generalized electrified disk problem for $w_{c}$ replaces $(2.15)$ :

$$
\partial_{\eta} w_{c}=0 \quad \text { on } \quad \eta=0, \quad\left(s_{1}, s_{2}\right) \notin \Omega ; \quad w_{c}=1 \quad \text { on } \quad \eta=0, \quad\left(s_{1}, s_{2}\right) \in \Omega,
$$

$$
w_{c} \sim c_{j} / \rho \text { as } \rho \rightarrow \infty \text {. }
$$

Here the absorbing set $\Omega$ in the plane $\eta=0$ is possibly multiconnected, which can incorporate the window clustering effect of [20]. When $\Omega$ has two lines of symmetry, then $w_{c}$ can be chosen to be even in $s_{1}$ and $s_{2}$, so that the far-field behavior of $w_{c}$ is $w_{c} \sim c_{j} / \rho+\mathcal{O}\left(\rho^{-3}\right)$ as $\rho \rightarrow \infty$, similar to that in (2.17). The analysis leading to (2.44) can then be repeated, and as remarked following (B.13) of Appendix B, the far-field behavior (2.37) will still hold for this generalized problem. Consequently, to treat the case of arbitrarily shaped absorbing windows centered at $x_{j}$ for $j=1, \ldots, N$ on the boundary of the unit sphere, we need only replace $c_{j}$ in (2.44) with the capacitance $c_{j}$ associated with the far-field behavior of (2.52). In addition, for an arbitrarily shaped absorbing window, we must also recalculate the monopole coefficient $\kappa_{j}$ in (2.44) from the solution to (B.4) subject to the far-field behavior (B.13). Although $c_{j}$ and $\kappa_{j}$ must 
in general be calculated numerically, such as from fast boundary integral methods of potential theory (cf. [46]), the capacitance $c_{j}$ is in fact known analytically for a few special geometries. In particular, for an elliptical-shaped absorbing window with semiaxes $a_{j}$ and $b_{j}$ so that $s_{1}^{2} / a_{j}^{2}+s_{2}^{2} / b_{j}^{2}=1$ with $b_{j}<a_{j}$, then $c_{j}$ is given in terms of the complete elliptic integral of the first kind $K(\mu)$ as (see [41])

$$
c_{j}=\frac{a_{j}}{K\left(e_{j}\right)}, \quad K(\mu)=\int_{0}^{\pi / 2} \frac{d \theta}{\sqrt{1-\mu^{2} \sin ^{2} \theta}}, \quad e_{j} \equiv \sqrt{1-b_{j}^{2} / a_{j}^{2}} .
$$

In addition, for the case of a cluster of two circular windows, each with radius $a_{j}$, and with center-to-center separation $2 l$, with $l>a_{j}$, it is known from [37] (see also equation (27) of [13]) that for $l / a_{j} \gg 1$

$$
c_{j} \sim \frac{4 a_{j}}{\pi}\left[1-\frac{a_{j}}{l \pi}+\frac{a_{j}^{2}}{l^{2} \pi^{2}}-\frac{a_{j}^{3}}{l^{3} \pi^{3}}+\cdots\right] .
$$

Upper and lower bounds for the capacitance $c_{j}$ of multiple nonoverlapping circular disks are derived in [13]. Similar bounds are used in [20] to study the effect of window clustering on the average MFPT, whereby several circular absorbing windows are clustered within an $\mathcal{O}(\varepsilon)$ region near some point on the boundary of the sphere. In our analysis, these bounds for the capacitance $c_{j}$ can be used in the three-term expansion (2.44) for the average MFPT.

3. The principal eigenvalue of the Laplacian. In this section we asymptotically calculate the principal eigenvalue of the Laplacian in the unit sphere $\Omega$, when the boundary of the sphere is almost entirely reflecting, but is perturbed by $N$ small nonoverlapping locally circular absorbing traps $\partial \Omega_{\varepsilon_{j}}$, centered at $x_{j}$ with $\left|x_{j}\right|=1$, for $j=1, \ldots, N$. The perturbed eigenvalue problem is

$$
\begin{aligned}
& \Delta u \equiv u_{r r}+\frac{2}{r} u_{r}+\frac{1}{r^{2} \sin ^{2} \theta} u_{\phi \phi}+\frac{\cot \theta}{r^{2}} u_{\theta}+\frac{1}{r^{2}} u_{\theta \theta}=-\lambda u, \quad x \in \Omega, \quad \int_{\Omega} u^{2} d x=1, \\
& \text { (3.1b) } \quad \partial_{r} u=0, \quad x \in \partial \Omega \backslash \partial \Omega_{a} ; \quad u=0, \quad x \in \partial \Omega_{a} \equiv \bigcup_{j=1}^{N} \partial \Omega_{\varepsilon_{j}} .
\end{aligned}
$$

Each boundary trap, $\partial \Omega_{\varepsilon_{j}}$, for $j=1, \ldots, N$ is a small "circular" cap centered at $\left(\theta_{j}, \phi_{j}\right)$, as defined by $(2.1 \mathrm{c})$, with area $\left|\partial \Omega_{\varepsilon_{j}}\right| \sim \pi \varepsilon^{2} a_{j}^{2}$, and with $\partial \Omega_{j} \rightarrow x_{j}$ as $\varepsilon \rightarrow 0$, where $x_{j}=\left(\cos \phi_{j} \sin \theta_{j}, \sin \phi_{j} \sin \theta_{j}, \cos \theta_{j}\right)$. Here $0 \leq \phi \leq 2 \pi$ is the longitude and $0 \leq \theta \leq \pi$ is the latitude.

We let $\lambda(\varepsilon)$ denote the principal eigenvalue of (3.1), with corresponding eigenfunction $u(x, \varepsilon)$. Clearly, $\lambda(\varepsilon) \rightarrow 0$ as $\varepsilon \rightarrow 0$ with $u \rightarrow u_{0}=|\Omega|^{-1 / 2}$. A leading-order calculation for $\lambda(\varepsilon)$ was given in section 5.2 of [47]. Here, we use a more refined matched asymptotic analysis to calculate a three-term asymptotic expansion for $\lambda(\varepsilon)$ as $\varepsilon \rightarrow 0$. Our asymptotic calculation will show, to within the three-term asymptotic approximation, that $\lambda(\varepsilon)$ is related to the average MFPT $\bar{v}$ of $(1.2)$ by $\lambda \sim 1 /(D \bar{v})$.

We expand the principal eigenvalue for (3.1) as

$$
\lambda=\varepsilon \lambda_{1}+\varepsilon^{2} \log \left(\frac{\varepsilon}{2}\right) \lambda_{2}+\varepsilon^{2} \lambda_{3}+\cdots .
$$

In the outer region away from the boundary traps we expand the outer solution as

$$
u \sim u_{0}+\varepsilon u_{1}+\varepsilon^{2} \log \left(\frac{\varepsilon}{2}\right) u_{2}+\varepsilon^{2} u_{3}+\cdots,
$$


where $u_{0} \equiv|\Omega|^{-1 / 2}$. The logarithmic terms in (3.2) and (3.3) arise as a direct consequence of the subdominant logarithmic singularity of the surface Neumann Green's function given in (2.7).

We first substitute (3.3) into (3.1) to obtain that $u_{k}$ for $k=1,2$ satisfies

$$
\Delta u_{k}=-\lambda_{k} u_{0}, \quad x \in \Omega ; \quad \partial_{r} u_{k}=0, \quad x \in \partial \Omega \backslash\left\{x_{1}, \ldots, x_{N}\right\} ; \quad \int_{\Omega} u_{k} d x=0 .
$$

In contrast, $u_{3}$ satisfies

$$
\begin{gathered}
\Delta u_{3}=-\lambda_{1} u_{1}-\lambda_{3} u_{0}, \quad x \in \Omega ; \quad \partial_{r} u_{3}=0, \quad x \in \partial \Omega \backslash\left\{x_{1}, \ldots, x_{N}\right\} ; \\
\int_{\Omega}\left(u_{1}^{2}+2 u_{0} u_{3}\right) d x=0 .
\end{gathered}
$$

In the inner region near the $j$ th boundary trap we introduce the local coordinate system $\left(\eta, s_{1}, s_{2}\right)$ by $(2.5)$. We then write the inner expansion of the principal eigenfunction as

$$
u \sim w_{0}+\varepsilon \log \left(\frac{\varepsilon}{2}\right) w_{1}+\varepsilon w_{2}+\cdots
$$

We substitute (3.6) into (3.1) and then transform the Laplacian in (3.1) in terms of the local coordinate system (2.5). Upon collecting similar terms in $\varepsilon$, we obtain that $w_{k}$ for $k=0,1,2$ satisfies $(2.13)$.

The leading-order matching condition is that $w_{0} \sim u_{0}=|\Omega|^{-1 / 2}$ as $\rho \equiv\left(\eta^{2}+\right.$ $\left.s_{1}^{2}+s_{2}^{2}\right)^{1 / 2} \rightarrow \infty$. Thus, we write

$$
w_{0}=u_{0}\left(1-w_{c}\right),
$$

where $w_{c}$ is the solution to the electrified disk problem (2.15), as given in (2.16). The far-field behavior of $w_{0}$ is given in (2.18). Upon writing (2.18) in terms of outer variables by using (2.5), we obtain the matching condition that the near-field behavior of the outer expansion (3.3) must agree with the far-field behavior of the inner expansion (3.6), so that

$$
u_{0}+\varepsilon u_{1}+\varepsilon^{2} \log \left(\frac{\varepsilon}{2}\right) u_{2}+\varepsilon^{2} u_{3}+\cdots \sim u_{0}\left(1-\frac{c_{j} \varepsilon}{\left|x-x_{j}\right|}+\cdots\right)+\varepsilon \log \left(\frac{\varepsilon}{2}\right) w_{1}+\varepsilon w_{2}+\cdots .
$$

From this matching condition, we obtain that $u_{1}$ must satisfy (3.4) and have the singular behavior $u_{1} \sim-c_{j} u_{0} /\left|x-x_{j}\right|$ as $x \rightarrow x_{j}$ for $j=1, \ldots, N$. This problem can be written in distributional form as

$$
\Delta u_{1}=-\lambda_{1} u_{0}, \quad x \in \Omega ;\left.\quad \partial_{r} u_{1}\right|_{r=1}=-2 \pi u_{0} \sum_{j=1}^{N} \frac{c_{j}}{\sin \theta_{j}} \delta\left(\theta-\theta_{j}\right) \delta\left(\phi-\phi_{j}\right),
$$

with $\int_{\Omega} u_{1} d x=0$. From the divergence theorem and by using $u_{0}=|\Omega|^{-1 / 2}$, we calculate $\lambda_{1}$ as

$$
\lambda_{1}=\frac{2 \pi}{|\Omega|} \sum_{j=1}^{N} c_{j}, \quad c_{j}=\frac{2 a_{j}}{\pi} .
$$

Copyright $@$ by SIAM. Unauthorized reproduction of this article is prohibited. 
Then, the solution to (3.9) with $\int_{\Omega} u_{1} d x=0$ can be written as

$$
u_{1}=-2 \pi u_{0} \sum_{i=1}^{N} c_{i} G_{s}\left(x ; x_{i}\right) .
$$

Here $G_{s}\left(x ; x_{j}\right)$ is the surface Neumann Green's function satisfying $(2.2)$. It is given explicitly in (2.4) and has the near-field behavior for $x \rightarrow x_{j}$ as given in (2.9).

Next, we expand $u_{1}$ in (3.11) as $x \rightarrow x_{j}$ by using the near-field behavior (2.9) for $G_{s}$. Upon substituting the resulting expression into the matching condition (3.8), we obtain

$$
\begin{aligned}
& u_{0}\left(1-\frac{c_{j}}{\rho}\right)+\frac{\varepsilon u_{0} c_{j}}{2} \log \left(\frac{\varepsilon}{2}\right)-2 \varepsilon \pi u_{0} B_{j} \\
& \quad+\frac{\varepsilon u_{0} c_{j}}{2}\left[\log (\eta+\rho)-\frac{\eta\left(s_{1}^{2}+s_{2}^{2}\right)}{\rho^{3}}+\frac{s_{1}^{2} s_{2} \cot \theta_{j}}{\rho^{3}}\right] \\
& \quad+\varepsilon^{2} \log \left(\frac{\varepsilon}{2}\right) u_{2}+\varepsilon^{2} u_{3}+\cdots \sim u_{0}\left(1-\frac{c_{j}}{\rho}\right)+\varepsilon \log \left(\frac{\varepsilon}{2}\right) w_{1}+\varepsilon w_{2}+\cdots
\end{aligned}
$$

Here the constant $B_{j}$ is defined by

$$
B_{j}=-\frac{9}{20 \pi} c_{j}+\sum_{\substack{i=1 \\ i \neq j}}^{N} c_{i} G_{s j, i}, \quad G_{s j, i} \equiv G_{s}\left(x_{j} ; x_{i}\right) .
$$

The matching condition (3.12) for the $\mathcal{O}(\varepsilon \log [\varepsilon / 2])$ terms yields that $w_{1} \sim c_{j} u_{0} / 2$ as $\rho \rightarrow \infty$. The solution $w_{1}$ to (2.13) is given in terms of the solution $w_{c}$ to $(2.15)$ with far-field behavior (2.18), so that

$$
w_{1}=\frac{c_{j} u_{0}}{2}\left(1-w_{c}\right) \sim \frac{c_{j} u_{0}}{2}\left(1-\frac{c_{j}}{\rho}+\mathcal{O}\left(\rho^{-3}\right)\right) \quad \text { as } \quad \rho \rightarrow \infty .
$$

Next, we substitute (3.14) into the matching condition (3.12) and use $\rho \sim \varepsilon^{-1} \mid x-$ $x_{0} \mid$. By matching the $\mathcal{O}\left(\varepsilon^{2} \log \varepsilon\right)$ terms, we obtain that $u_{2}$ satisfies (3.4) with singular behavior $u_{2} \sim-c_{j}^{2} u_{0} /\left(2\left|x-x_{j}\right|\right)$ as $x \rightarrow x_{j}$ for $j=1, \ldots, N$. Therefore, in distributional form, the problem for $u_{2}$ is equivalent to

$$
\Delta u_{2}=-\lambda_{2} u_{0}, \quad x \in \Omega ;\left.\quad \partial_{r} u_{2}\right|_{r=1}=-\pi u_{0} \sum_{j=1}^{N} c_{j}^{2} \frac{\delta\left(\theta-\theta_{j}\right) \delta\left(\phi-\phi_{j}\right)}{\sin \theta_{j}},
$$

with $\int_{\Omega} u_{2} d x=0$. From the divergence theorem and by using $u_{0}=|\Omega|^{-1 / 2}$, we calculate $\lambda_{2}$ as

$$
\lambda_{2}=\frac{\pi}{|\Omega|} \sum_{j=1}^{N} c_{j}^{2}
$$

Then, the solution $u_{2}$ to (3.15), with $\int_{\Omega} u_{2} d x=0$, is written in terms of $G_{s}\left(x ; x_{j}\right)$ as

$$
u_{2}=-\pi u_{0} \sum_{j=1}^{N} c_{j}^{2} G_{s}\left(x ; x_{j}\right)
$$

Copyright (c) by SIAM. Unauthorized reproduction of this article is prohibited. 
Next, we match the $\mathcal{O}(\varepsilon)$ terms on the left-hand side of (3.12). We obtain that $w_{2}$ satisfies the inhomogeneous problem (2.13) and has the far-field behavior (3.18)

$$
w_{2} \sim-2 \pi u_{0} B_{j}+\frac{c_{j} u_{0}}{2}\left[\log (\eta+\rho)-\frac{\eta\left(s_{1}^{2}+s_{2}^{2}\right)}{\rho^{3}}+\frac{s_{1}^{2} s_{2} \cot \theta_{j}}{\rho^{3}}\right] \quad \text { as } \quad \rho \rightarrow \infty .
$$

To determine $w_{2}$, we first set $w_{0}=u_{0}\left(1-w_{c}\right)$ in the definition of the inhomogeneous term $\mathcal{F}_{2}$ in $(2.13 \mathrm{c})$, where $w_{c}$ is the solution to (2.15). Then, we decompose $w_{2}$ into the sum of three terms as

$$
w_{2}=-2 \pi B_{j} u_{0}\left(1-w_{c}\right)+u_{0} w_{2 e}+u_{0} w_{2 o} .
$$

With the operator $\mathcal{L}$ as defined in (2.13a), $w_{2 e}$ and $w_{2 o}$ are the solutions of (2.33) and (2.34), respectively.

The explicit solution to (2.33) for $w_{2 e}$ is given in Lemma B.1 of Appendix B. This solution has the far-field behavior (2.35). The explicit solution to (2.34) for $w_{2 o}$ is given by Lemma B.2 of Appendix B. In this way, we obtain that $w_{2}$ has the far-field behavior

$$
\begin{aligned}
w_{2} \sim & -2 \pi B_{j} u_{0}\left(1-\frac{c_{j}}{\rho}\right)+\frac{c_{j} u_{0}}{2}\left[\log (\eta+\rho)-\frac{\eta}{\rho^{3}}\left(s_{1}^{2}+s_{2}^{2}\right)-\frac{2 \kappa_{j}}{\rho}\right] \\
& -\frac{c_{j} \eta}{2 \rho^{3}}\left(s_{1}^{2}+s_{2}^{2}\right)+o\left(\rho^{-1}\right) \quad \text { as } \quad \rho \rightarrow \infty .
\end{aligned}
$$

By substituting (3.20) into the matching condition (3.12), we obtain that the two monopole terms in (3.20) proportional to $\rho^{-1}$ determine the singularity behavior for the correction term $u_{3}$ in (3.12). Therefore, we obtain that $u_{3}$ satisfies (3.5) with the singular behavior

$$
u_{3} \sim \frac{2 \pi B_{j} c_{j} u_{0}}{\left|x-x_{j}\right|}-\frac{c_{j} u_{0} \kappa_{j}}{\left|x-x_{j}\right|} \quad \text { as } \quad x \rightarrow x_{j}, \quad j=1, \ldots, N,
$$

where $\kappa_{j}$ is defined in (2.36). This problem for $u_{3}$ can be written in distributional form as

$$
\begin{aligned}
\Delta u_{3} & =-\lambda_{1} u_{1}-\lambda_{3} u_{0}, \quad x \in \Omega ; \\
\left.\partial_{r} u_{3}\right|_{r=1} & =u_{0} \sum_{j=1}^{N}\left(4 \pi^{2} B_{j} c_{j}-2 \pi c_{j} \kappa_{j}\right) \frac{\delta\left(\theta-\theta_{j}\right) \delta\left(\phi-\phi_{j}\right)}{\sin \theta_{j}} .
\end{aligned}
$$

By using the divergence theorem, together with $u_{0}=|\Omega|^{-1 / 2}, \int_{\Omega} u_{1} d x=0$, and (2.36), we calculate $\lambda_{3}$ as

$$
\lambda_{3}=-\frac{4 \pi^{2}}{|\Omega|} \sum_{j=1}^{N} B_{j} c_{j}+\frac{\pi}{|\Omega|} \sum_{j=1}^{N} c_{j}^{2}\left(2 \log 2-\frac{3}{2}+\log a_{j}\right) .
$$

Finally, we substitute $u_{0}=|\Omega|^{-1 / 2},(3.11)$, and (3.17) into (3.3) to obtain the outer expansion of the eigenfunction. The perturbed eigenvalue is obtained by substituting (3.10), (3.16), and (3.23) into (3.2). We summarize the result as follows.

Principal Result 3.1. Consider (3.1) in the unit sphere $\Omega$ with $N$ small circular boundary traps of radius $\varepsilon a_{j}$ on $\partial \Omega$ centered at $x_{j}$ for $j=1, \ldots, N$. Then, for 
$\varepsilon \rightarrow 0$, the asymptotic solution to (3.1) is given in the outer region $\left|x-x_{j}\right| \gg \mathcal{O}(\varepsilon)$ for $j=1, \ldots, N$ by

$$
u=\frac{1}{|\Omega|^{1 / 2}}\left(1-2 \pi \varepsilon \sum_{j=1}^{N} c_{j} G_{s}\left(x ; x_{j}\right)-\varepsilon^{2} \pi \log \left(\frac{\varepsilon}{2}\right) \sum_{j=1}^{N} c_{j}^{2} G_{s}\left(x ; x_{j}\right)+\mathcal{O}\left(\varepsilon^{2}\right)\right) .
$$

Here $c_{j}=2 a_{j} / \pi$ is the capacitance associated with the $j$ th boundary trap of radius $\varepsilon a_{j}$, and $G_{s}\left(x ; x_{j}\right)$ is the surface Neumann Green's function given in (2.4). For $\varepsilon \rightarrow 0$, the principal eigenvalue $\lambda(\varepsilon)$ of $(3.1)$ is given by

$\lambda=\frac{2 \pi \varepsilon N \bar{c}}{|\Omega|}+\frac{\pi \varepsilon^{2}}{|\Omega|} \sum_{j=1}^{N} c_{j}^{2}\left[\log \left(\varepsilon a_{j}\right)+\log 2-\frac{3}{2}\right]-\frac{4 \pi^{2} \varepsilon^{2}}{|\Omega|} p_{c}\left(x_{1}, \ldots, x_{N}\right)+\mathcal{O}\left(\varepsilon^{3} \log \varepsilon\right)$,

where $\bar{c} \equiv N^{-1}\left(c_{1}+\cdots+c_{N}\right)$. The quadratic form $p_{c}\left(x_{1}, \ldots, x_{N}\right)$ in (3.25a) is defined in terms of the entries $\mathcal{G}_{s i, j}$ of the Green's matrix $\mathcal{G}_{s}$ of (2.42) by the weighted discrete sum

$$
p_{c}\left(x_{1}, \ldots, x_{N}\right) \equiv \sum_{i=1}^{N} \sum_{j=1}^{N} c_{i} c_{j} \mathcal{G}_{s i, j}, \quad c_{j}=\frac{2 a_{j}}{\pi},
$$

where $\mathcal{G}_{s i, j} \equiv G_{s}\left(x_{i} ; x_{j}\right)$ is given explicitly from (2.4) by

$$
\begin{gathered}
\mathcal{G}_{s i, j}=-\frac{9}{20 \pi}+\frac{\left(1-\delta_{i j}\right)}{2 \pi}\left[\log 2+\mathcal{H}_{s i, j}\right], \quad i, j=1, \ldots, N, \\
\mathcal{H}_{s i, j}=\frac{1}{\left|x_{i}-x_{j}\right|}-\frac{1}{2} \log \left|x_{i}-x_{j}\right|-\frac{1}{2} \log \left(2+\left|x_{i}-x_{j}\right|\right), \quad i \neq j .
\end{gathered}
$$

Here $\delta_{i j}=1$ if $i=j$ and $\delta_{i j}=0$ if $i \neq j$. In terms of $\mathcal{H}_{s i, j}$, we can write (3.25a) as

$$
\begin{aligned}
& \lambda=\frac{2 \pi \varepsilon N \bar{c}}{|\Omega|}+\frac{\pi \varepsilon^{2}}{|\Omega|} \sum_{j=1}^{N} c_{j}^{2}\left[\log \left(\varepsilon a_{j}\right)+3 \log 2-\frac{3}{2}\right] \\
& -\frac{4 \pi^{2} \varepsilon^{2}}{|\Omega|} \sum_{i=1}^{N} \sum_{j=i+1}^{N} c_{i} c_{j} \mathcal{H}_{s i, j}+\frac{\pi \varepsilon^{2}}{|\Omega|}\left(\frac{9}{5}-2 \log 2\right) \sum_{i=1}^{N} \sum_{j=1}^{N} c_{i} c_{j}+\mathcal{O}\left(\varepsilon^{3} \log \varepsilon\right) .
\end{aligned}
$$

For the special case where there are $N$ identical circular boundary traps of a common radius $\varepsilon a$, then (3.25e) with $c=2 a / \pi$ and $|\Omega|=4 \pi / 3$ reduces to

$$
\begin{aligned}
\lambda \sim \frac{2 \pi \varepsilon N c}{|\Omega|}[1 & +\frac{\varepsilon c}{2} \log (\varepsilon a)+\frac{\varepsilon c}{2}\left(\log 2-\frac{3}{2}\right)+\frac{9 \varepsilon c N}{10} \\
& \left.-\varepsilon c(N-1) \log 2-\frac{2 \varepsilon c}{N} \mathcal{H}\left(x_{1}, \ldots, x_{N}\right)\right] .
\end{aligned}
$$

Here the discrete energy function $\mathcal{H}\left(x_{1}, \ldots, x_{N}\right) \equiv \sum_{i=1}^{N} \sum_{j=i+1}^{N} \mathcal{H}_{s i, j}$ is given by (2.51b). For the case of one single trap, the result (3.26) yields the three-term expansion

$$
\lambda \sim \frac{2 \pi \varepsilon c}{|\Omega|}\left[1+\frac{\varepsilon c}{2}\left(\log (\varepsilon a)+\log 2-\frac{3}{2}\right)+\frac{9 \varepsilon c}{10}\right] .
$$

Copyright ( $)$ by SIAM. Unauthorized reproduction of this article is prohibited. 
Upon setting $c=2 / \pi$ in (3.26), corresponding to $N$ locally circular traps of a common radius $\varepsilon$, it is readily verified from (3.26) and (2.51) that, to within the threeterm asymptotic approximations, the relation $\bar{v} \sim 1 /(D \lambda)$ between the average MFPT and the principal eigenvalue is asymptotically valid in the limit $\varepsilon \rightarrow 0$. Therefore, we conclude that for the case of $N$ circular boundary traps of a common radius $\varepsilon a$ the principal eigenvalue of (3.1) is maximized, and the corresponding MFPT minimized, in the limit $\varepsilon \rightarrow 0$ at the boundary trap configuration $\left\{x_{1}, \ldots, x_{N}\right\}$ that minimizes the discrete sum $\mathcal{H}\left(x_{1}, \ldots, x_{N}\right)$ in $(2.51 \mathrm{~b})$ on the unit sphere $\left|x_{j}\right|=1$ for $j=1, \ldots, N$.

4. Numerical optimization results for the unit sphere. Next, we numerically compute the optimum energy and the optimum arrangements $\left\{x_{1}, \ldots, x_{N}\right\}$ of the centers of $N \geq 3$ circular boundary traps of a common radius that minimize the discrete energy (2.51b). We compare our results with corresponding results associated with minimizing either the Coulomb energy or the logarithmic energy defined by

$$
\mathcal{H}_{\mathrm{C}}=\sum_{i=1}^{N} \sum_{j=i+1}^{N} \frac{1}{\left|x_{i}-x_{j}\right|}, \quad \mathcal{H}_{\mathrm{L}}=-\sum_{i=1}^{N} \sum_{j=i+1}^{N} \log \left|x_{i}-x_{j}\right|
$$

Various numerical methods for global optimization are available (cf. [31], [21], [36]), including methods for nonsmooth optimization and optimization with constraints. For low-dimensional problems, exact methods are available, whereas for higher-dimensional problems one often must use heuristic strategies, including evolution algorithms and simulated annealing. The following methods were used to confirm our numerical optimization results for (2.51b):

1. The extended cutting angle method (ECAM). This deterministic global optimization technique is applicable to Lipschitz functions. Within the algorithm, a sequence of piecewise linear lower approximations to the objective function is constructed. The sequence of the corresponding solutions to these relaxed problems converges to the global minimum of the objective function (cf. [1]).

2. Dynamical systems based optimization (DSO). A dynamical system is constructed, using a number of sampled values of the objective function to introduce "forces." The evolution of such a system yields a descent trajectory converging to lower values of the objective function. The algorithm continues sampling the domain until it converges to a stationary point (cf. [29]).

3. Lipschitz-continuous global optimizer (LGO). This is a commercial global optimization software program available for a number of software and hardware platforms, based on a combination of several rigorous (theoretically convergent) global minimization strategies, as well as a number of local minimization strategies. For further details, see [31].

On a unit sphere, it is convenient to write the location $x_{j}$ of each trap in terms of spherical coordinates $\left(\theta_{j}, \phi_{j}\right)$, where $\theta_{j}$ is the latitude and $\phi_{j}$ is the longitude. To partially eliminate the effect of the rotational symmetries of the sphere, we fix the first trap $x_{1}$ at the north pole, i.e., $\left(\theta_{1}, \phi_{1}\right)=(0,0)$, and we let $\phi_{2}=0$ for the second trap centered at $x_{2}$. Then, for $N$ traps on the unit sphere, one has a global optimization problem of $2 N-3$ parameters in the range $0<\theta_{j} \leq \pi$ for $j=2, \ldots, N$ and $0 \leq \phi_{j}<2 \pi$ for $j=3, \ldots, N$. As an "initial guess" for the global optimization routines, we chose the remaining traps $x_{2}, \ldots, x_{N}$ to be equally spaced on the equator $\theta=\pi / 2$.

For $3 \leq N \leq 20$ traps, the ECAM and DSO methods, as outlined above and implemented in the open software library GANSO [14], were used to obtain the numerical 
TABLE 4.1

Numerically computed minimal values of the discrete energy $\mathcal{H}$ of $(2.51 \mathrm{~b})$ for $3 \leq N \leq 20$, together with the optimal Coulomb and logarithmic energies in (4.1) for $N$-trap arrangements on the unit sphere. The results were obtained using both the ECAM and the DSO methods and are believed to be correct to the number of digits shown.

\begin{tabular}{|c|c|c|c|}
\hline$N$ & $\mathcal{H}(2.51 \mathrm{~b})$ & $\mathcal{H}_{\mathrm{C}}(4.1)$ & $\mathcal{H}_{\mathrm{L}}(4.1)$ \\
& ECAM (DSO) & ECAM (DSO) [Refs. [18], [34]] & ECAM (DSO) [Ref. [3]] \\
\hline 3 & $-1.067345(-1.067345)$ & $1.732051(1.732051)[-]$ & $-1.647918(-1.647918)$ \\
4 & $-1.667180(-1.667180)$ & $3.674234(3.674234)[3.674234]$ & $-2.942488(-2.942488)$ \\
5 & $-2.087988(-2.087988)$ & $6.474691(6.474691)[6.474692]$ & $-4.420507(-4.420507)$ \\
6 & $-2.581006(-2.581006)$ & $9.985281(9.985281)[9.985281]$ & $-6.238324(-6.238324)$ \\
7 & $-2.763658(-2.763658)$ & $14.452978(14.452977)[14.452977]$ & $-8.182476(-8.182477)$ \\
8 & $-2.949577(-2.949576)$ & $19.675288(19.675288)[19.675288]$ & $-10.428018(-10.428018)$ \\
9 & $-2.976434(-2.976434)$ & $25.759987(25.759987)[25.759987]$ & $-12.887753(-12.887753)$ \\
10 & $-2.835735(-2.835735)$ & $32.716950(32.716950)[32.716950]$ & $-15.563123(-15.563123)$ \\
11 & $-2.456734(-2.456734)$ & $40.596450(40.596522)[40.596451]$ & $-18.420480(-18.420480)$ \\
12 & $-2.161284(-2.161284)$ & $49.165253(49.165253)[49.165253]$ & $-21.606145(-21.606145)$ \\
16 & $1.678405(1.678405)$ & $92.911655(92.911655)[92.911655]$ & $-36.106152(-36.106152)$ \\
20 & $8.481790(8.481790)$ & $150.881571(150.881569)[150.881568]$ & $-54.011130(-54.011130)$ \\
\hline
\end{tabular}

results in Table 4.1 for the global minimum of the discrete energy (2.51b) and the two classic energies of (4.1). A good agreement between the ECAM and DSO methods for the minimum values of these three discrete energy functions, as well as the optimal trap locations, were used to validate the results. For the classic discrete energies in (4.1), our results compare favorably with the tabulated data of [18], [11], and [34] for the Coulomb energy and with the results of [3] for the logarithmic energy. From Table 4.1, it is interesting to observe that for these values of $N$ the minimal Coulomb and logarithmic energies are monotone functions of $N$, whereas (2.51b) has a local minimum for $N=9$ traps. Due to the difficulty in trying to find optimal trap arrangements analytically, we are unable to offer a theoretical explanation for this observation here. By using numerical optimization LGO software, further data for the optimal values of $\mathcal{H}$ for larger values of $N$ were computed by Prof. Raymond Spiteri and Sheldon Richards [45], and these are given in Table 4.2.

For $N$ circular traps of a common radius $\varepsilon$, the average MFPT $\bar{v}$ is given in (2.51), and the corresponding principal eigenvalue $\lambda$ is obtained upon setting $a=1$ and $c=2 / \pi$ in (3.26). We then use the results for the optimum value of $\mathcal{H}$ as given in Table 4.2 to show the significant effect on the optimal $\bar{v}$ and $\lambda$ of the fragmentation of the trap set on the surface of the unit sphere. More specifically, to study the effect of fragmentation, we denote the percentage surface area fraction of traps by $100 f$, where $f \equiv N \pi \varepsilon^{2} / 4 \pi=N \varepsilon^{2} / 4$. Then, for each fixed value of $100 f$, where $f$ is small, in Figure 4.1(a) we plot $\bar{v}$ versus $100 f$ for the optimal arrangement of $N=5,10,20,30,40,50,60$ traps on the surface of the unit sphere. In this figure we also plot $\bar{v}$ for a single large trap having the same trap surface area fraction. In Figure 4.1(b) we plot the corresponding optimal value for the principal eigenvalue versus $100 f$. For $N$ not too large, we conclude that even when $f$ is small the effect of fragmentation of the trap set is rather significant. The clustering of the curves in Figure 4.1(a) when $N$ becomes larger suggests that the effect of fragmentation decreases significantly when the traps are sufficiently dispersed over the surface of the sphere.

To further illustrate our asymptotic results, we take $N=11$ locally circular traps 
TABLE 4.2

Numerically computed minimal values of the discrete energy $\mathcal{H}$ of (2.51b) for $N$-trap arrangements on the unit sphere. The results were obtained by Prof. Raymond Spiteri and Sheldon Richards [45] using a global optimization package based on the LGO method (see [31]) and are believed to be correct to the number of digits shown.

\begin{tabular}{|c|c|c|c|c|c|}
\hline$N$ & $\mathcal{H}$ & $N$ & $\mathcal{H}$ & $N$ & $\mathcal{H}$ \\
\hline 3 & -1.067345 & 24 & 18.5819814721 & 45 & 130.9053155742 \\
4 & -1.6671798784 & 25 & 21.7249125077 & 46 & 138.9204719381 \\
5 & -2.0879876408 & 26 & 25.0100311587 & 47 & 147.1503517898 \\
6 & -2.5810055438 & 27 & 28.4296992470 & 48 & 155.4174212367 \\
7 & -2.7636583659 & 28 & 32.1929330003 & 49 & 164.2174643572 \\
8 & -2.9495765146 & 29 & 36.2197825589 & 50 & 173.0786752357 \\
9 & -2.9764336362 & 30 & 40.3544394110 & 51 & 182.2666361905 \\
10 & -2.8357352067 & 31 & 44.7576166539 & 52 & 191.7242795403 \\
11 & -2.4567341080 & 32 & 49.2409493909 & 53 & 201.3847502086 \\
12 & -2.1612842350 & 33 & 54.2959715142 & 54 & 211.2834896820 \\
13 & -1.3678268562 & 34 & 59.3794884966 & 55 & 221.4638143503 \\
14 & -0.5525927824 & 35 & 64.7367106977 & 56 & 231.8539761252 \\
15 & 0.4774375981 & 36 & 70.2760966504 & 57 & 242.5180260656 \\
16 & 1.6784048848 & 37 & 76.0662374477 & 58 & 253.4345991848 \\
17 & 3.0751594437 & 38 & 82.0802998438 & 59 & 264.5718557147 \\
18 & 4.6651247247 & 39 & 88.3295602222 & 60 & 275.9094168850 \\
19 & 6.5461713534 & 40 & 94.8178306425 & 61 & 287.6211395726 \\
20 & 8.4817895578 & 41 & 101.5685414461 & 62 & 299.4803102551 \\
21 & 10.7013196449 & 42 & 108.5402790527 & 63 & 311.6558516852 \\
22 & 13.1017418136 & 43 & 115.7702835060 & 64 & 324.0896310134 \\
23 & 15.8212820967 & 44 & 123.1634320345 & 65 & 336.7697097130 \\
\hline
\end{tabular}

of a common radius $\varepsilon$ and in Figure $4.2(\mathrm{a})$ we plot $\bar{v}$ in (2.51) versus $\varepsilon$ for three different point arrangements on the sphere, including the set of points that minimize $\mathcal{H}$ in (2.51b). For $N=11$ and $\varepsilon=0.2$, the traps occupy $11 \%$ of the surface area of the sphere. The optimal point arrangement on the sphere is depicted in Figure 4.2(b). From Figure 4.2(a) we observe that a randomly generated point arrangement gives a result for $\bar{v}$ that is rather close to that for the optimal point arrangement. For $\varepsilon=0.1907$, the 11 traps occupy about $10 \%$ of the surface area of the sphere, and the optimal $\bar{v}$ is $\bar{v} \approx 0.368$. We remark that for a single large trap with a $10 \%$ surface area fraction its radius must be $\varepsilon=0.6325$. For this value of $\varepsilon,(2.45)$ yields $\bar{v} \approx 1.48$, which is about three times larger than for the optimal point arrangement. This example shows clearly the significant effect on $\bar{v}$ of trap fragmentation.

Next, we remark on the spatial configuration of the optimal arrangement of traps for small $N$. For $N=2,3,4$, the optimal trap arrangements for the discrete energy (2.51b) and the two classical energies of (4.1) must be the same, since equidistant spherical arrangements are available. For $N=2$, the traps occupy the two poles; for $N=3$, they are located at the vertices of an inscribed equilateral triangle; and for $N=4$, they are located at the vertices of an inscribed tetrahedron. Our computational results show that, for at least $2 \leq N \leq 20$, the optimal trap arrangements for the discrete energy (2.51b) and the two classical energies of (4.1) are the same. It is an open problem to prove this result and to investigate numerically whether this equivalence holds for even larger values of $N$.

In Table 4.3 we give our numerical results for the optimal trap locations when $3 \leq$ $N \leq 12$. The numerically computed minimal energy arrangements for $N=4,5,6,7$ are shown in Figure 4.3. For $N=5, N=6$, and $N=7$, two traps are located at the poles, while the other $N-2$ traps are on the equator. For $N=8,9,10,12$, the 


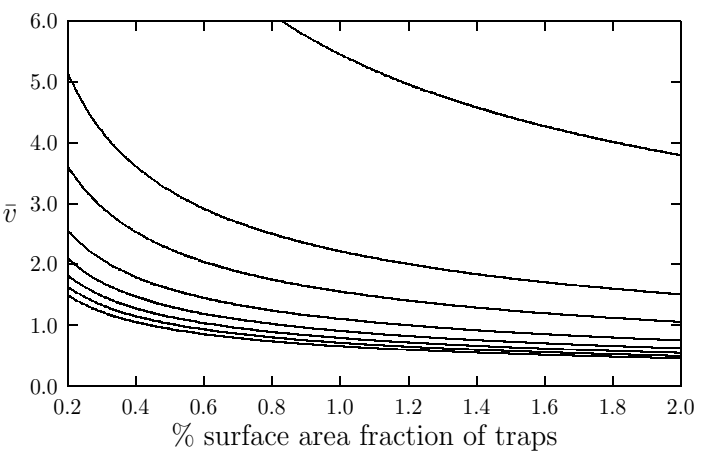

(a) $\bar{v}$ versus $f$

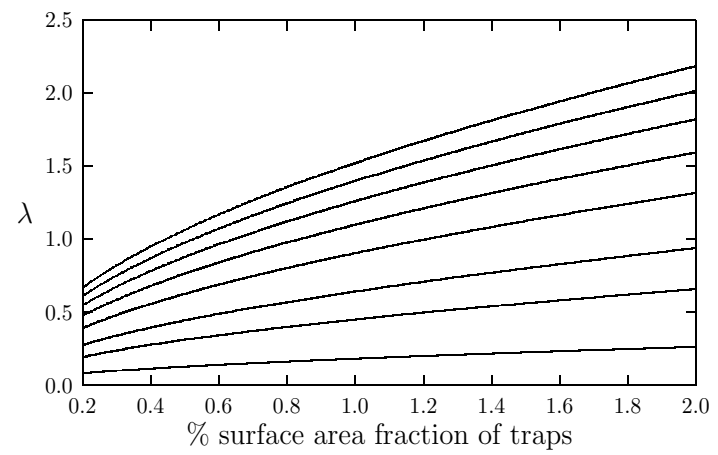

(b) $\lambda$ versus $f$

FIG. 4.1. The average MFPT $\bar{v}$ in (2.51) with $D=1$ and the principal eigenvalue $\lambda$ of (3.26) with $c=2 / \pi$ and $a=1$ versus the percentage trap surface area fraction $100 f$, where $f=N \varepsilon^{2} / 4$, for the optimal arrangement of $N$ identical circular traps of a common radius $\varepsilon$ on the boundary of the unit sphere. Top figure: $\bar{v}$ versus $100 \mathrm{f}$ for $N=1,5,10,20,30,40,50,60$ (top to bottom curves). Bottom figure: $\lambda$ versus $f$ for $N=1,5,10,20,30,40,50,60$ (bottom to top curves).

minimal energy arrangements are more irregular and are shown in Figure 4.4. For $N=10,12$, the minimal energy arrangements have two "belts" of traps with common latitude $\theta$, with two traps located at the poles.

Next, we formally derive a scaling law as $N \rightarrow \infty$ for the discrete energy $\mathcal{H}\left(x_{1}, \ldots\right.$, $\left.x_{N}\right)$ in $(2.51 \mathrm{~b})$. We decompose $\mathcal{H}$ into the sum of three terms as

$$
\mathcal{H}\left(x_{1}, \ldots, x_{N}\right)=\mathcal{H}_{1}+\mathcal{H}_{2}+\mathcal{H}_{3},
$$

where

$$
\begin{aligned}
\mathcal{H}_{1} & =\sum_{i=1}^{N} \sum_{j=i+1}^{N} \frac{1}{\left|x_{i}-x_{j}\right|}, \quad \mathcal{H}_{2}=-\frac{1}{2} \sum_{i=1}^{N} \sum_{j=i+1}^{N} \log \left|x_{i}-x_{j}\right|, \\
\mathcal{H}_{3} & =-\frac{1}{2} \sum_{i=1}^{N} \sum_{j=i+1}^{N} \log \left(2+\left|x_{i}-x_{j}\right|\right) .
\end{aligned}
$$

We then derive an approximation to the optimal value of $\mathcal{H}_{j}$ as $N \rightarrow \infty$ for $j=1,2,3$ by using the mean-field approximation method of [15] and [3]. 


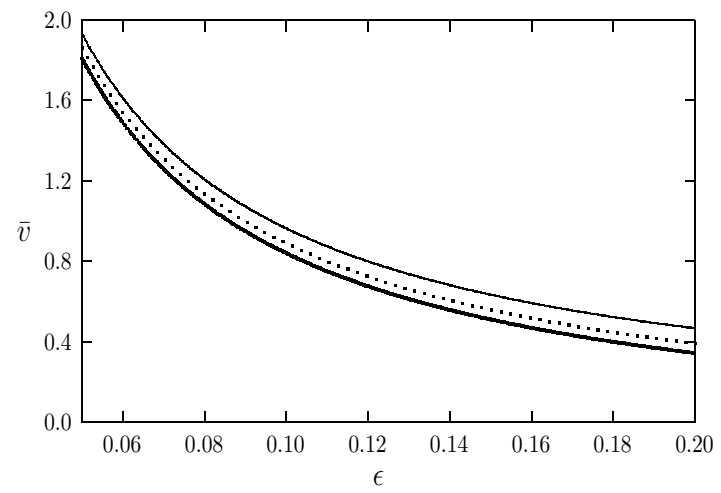

(a) $\bar{v} \operatorname{versus} \varepsilon$

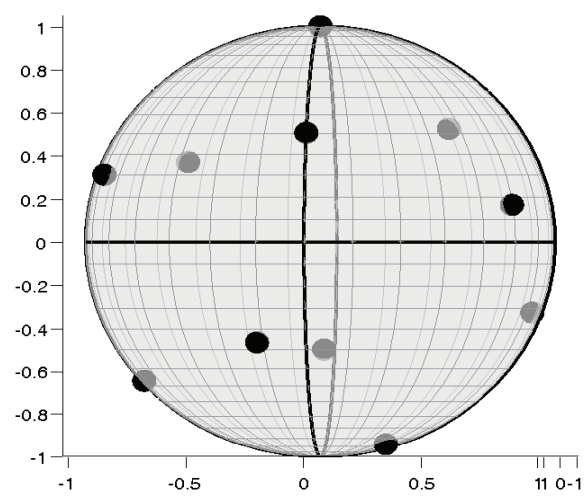

(b) Optimal points on the sphere

FIG. 4.2. Top figure: plot of the average MFPT $\bar{v}$ in (2.51) versus $\varepsilon$ for $D=1$ and $N=11$ and three different arrangements of points on the sphere. The heavy solid curve corresponds to the minimum point of $\mathcal{H}$ in $(2.51 \mathrm{~b})$, the solid curve is for 11 points equidistantly spaced on the equator, and the dotted curve is for a randomly generated point arrangement. Bottom figure: the optimal arrangement of $N=11$ points on the sphere that minimize $\mathcal{H}$ in (2.51b).

We first consider the Coulomb term $\mathcal{H}_{1}$, as was discussed in [15]. Suppose that a charge is located at the north pole. We write its interaction energy $E_{1 i}$ with the $i$ th other charge as

$$
E_{1 i}^{(1)}=\frac{1}{r_{1 i}}, \quad r_{1 i}=\left|x_{1}-x_{i}\right|=\sqrt{2(1-\cos \theta)},
$$

where $\theta$ is the azimuthal angle of the particle located at $x_{i}$. For large $N$, we assume that the charges are distributed "homogeneously" on the sphere, and that there is no charge in the azimuthal neighborhood $0 \leq \theta<\theta_{0}$ of the north pole, where $\theta_{0} \ll 1$. Therefore, for $\theta_{0} \ll 1$, the number density of charges is given approximately by

$$
P(\theta, \phi)= \begin{cases}\frac{N}{4 \pi} & \text { for } \theta_{0}<\theta<\pi \\ 0 & \text { for } 0<\theta<\theta_{0}\end{cases}
$$

where $\theta_{0}$ is determined from the condition that $\int_{0}^{2 \pi} \int_{\theta_{0}}^{\pi} P(\theta, \phi) \sin \theta d \theta d \phi=N-1$, 
TABLE 4.3

Spherical coordinates $(\theta, \phi)$ of the optimal locations of $3 \leq N \leq 12$ traps. These arrangements simultaneously minimize the discrete energy (2.51b) and the two classical discrete energies in (4.1).

\begin{tabular}{|c|c|c|c|c|c|c|c|c|c|c|c|c|c|}
\hline$N$ & & \multicolumn{12}{|c|}{ Spherical coordinates of optimal trap locations } \\
\hline \multirow[t]{2}{*}{3} & $\theta$ & 0.000 & 2.094 & 2.094 & & & & & & & & & \\
\hline & $\phi$ & 0.000 & 0.000 & 3.142 & & & & & & & & & \\
\hline \multirow[t]{2}{*}{4} & $\bar{\theta}$ & 0.000 & 1.911 & 1.911 & 1.911 & & & & & & & & \\
\hline & $\phi$ & 0.000 & 0.000 & 2.094 & 4.189 & & & & & & & & \\
\hline \multirow[t]{2}{*}{5} & $\bar{\theta}$ & 0.000 & 1.571 & 1.571 & 1.571 & 3.142 & & & & & & & \\
\hline & $\phi$ & 0.000 & 0.000 & 2.094 & 4.189 & 0.000 & & & & & & & \\
\hline \multirow[t]{2}{*}{6} & $\theta$ & 0.000 & 1.571 & 1.571 & 1.571 & 1.571 & 3.142 & & & & & & \\
\hline & $\phi$ & 0.000 & 0.000 & 1.571 & 3.142 & 4.712 & 0.000 & & & & & & \\
\hline \multirow[t]{2}{*}{7} & $\theta$ & 0.000 & 1.570 & 1.570 & 1.570 & 1.570 & 1.570 & 3.142 & & & & & \\
\hline & $\phi$ & 0.000 & 0.000 & 2.513 & 5.027 & 1.257 & 3.770 & 0.000 & & & & & \\
\hline \multirow[t]{2}{*}{8} & $\theta$ & 0.000 & 1.251 & 1.251 & 1.399 & 1.399 & 1.952 & 2.497 & 2.497 & & & & \\
\hline & $\phi$ & 0.000 & 1.445 & 3.565 & 0.000 & 5.010 & 2.505 & 0.706 & 4.304 & & & & \\
\hline \multirow[t]{2}{*}{9} & $\theta$ & 0.000 & 1.207 & 1.207 & 1.325 & 1.325 & 1.561 & 2.361 & 2.415 & 2.415 & & & \\
\hline & $\phi$ & 0.000 & 0.000 & 2.369 & 3.639 & 5.013 & 1.185 & 4.326 & 2.369 & 0.000 & & & \\
\hline \multirow[t]{2}{*}{10} & $\theta$ & 0.000 & 1.134 & 1.134 & 1.134 & 1.134 & 2.007 & 2.007 & 2.007 & 2.007 & 3.142 & & \\
\hline & $\phi$ & 0.000 & 0.000 & 1.571 & 3.142 & 4.712 & 0.785 & 2.356 & 3.927 & 5.498 & 0.000 & & \\
\hline \multirow[t]{2}{*}{11} & $\theta$ & 0.000 & 1.041 & 1.019 & 1.192 & 1.254 & 1.399 & 1.906 & 2.095 & 2.056 & 2.272 & 2.799 & \\
\hline & $\phi$ & 0.000 & 0.000 & 2.516 & 3.862 & 5.047 & 1.041 & 1.948 & 3.194 & 6.044 & 4.576 & 1.042 & \\
\hline \multirow[t]{2}{*}{12} & $\bar{\theta}$ & 0.000 & 1.107 & 1.107 & 1.107 & 1.107 & 1.107 & 2.035 & 2.035 & 2.035 & 2.035 & 2.035 & 3.142 \\
\hline & $\phi$ & 0.000 & 0.628 & 1.885 & 3.142 & 4.398 & 5.655 & 0.000 & 1.257 & 2.513 & 3.770 & 5.026 & 2.132 \\
\hline
\end{tabular}

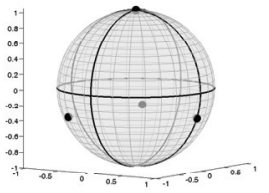

(a) $N=4$

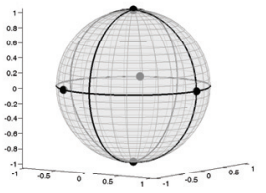

(b) $N=5$

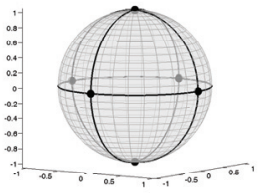

(c) $N=6$

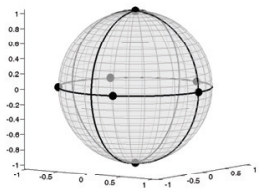

(d) $N=7$

FIG. 4.3. Minimal energy trap configurations for $N=4,5,6,7$ traps, common for the three discrete energy functions.

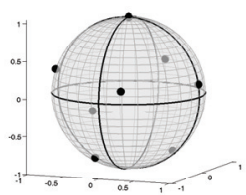

(a) $N=8$

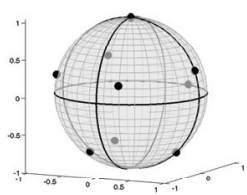

(b) $N=9$

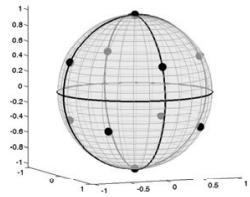

(c) $N=10$

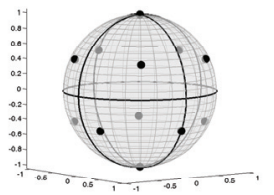

(d) $N=12$

FIG. 4.4. Minimal energy trap configurations for $N=8,9,10,12$ traps, common for the three discrete energy functions.

which yields $\cos \theta_{0}=1-2 / N$. For $N \gg 1$, we use $\cos \theta_{0} \approx 1-\theta_{0}^{2} / 2$, to obtain $\theta_{0} \approx \sqrt{4 / N}$, as was given in [3].

Next, the interaction energy of the north-pole charge with the remaining charges is approximated by

$$
\epsilon_{1}=\int_{0}^{2 \pi} \int_{\theta_{0}}^{\pi} P(\theta, \phi) E_{1 i}^{(1)} \sin \theta d \theta d \phi
$$

which can be calculated analytically as $\epsilon_{1}=-N\left[\sin \left(N^{-1 / 2}\right)-1\right]$. From a Taylor series expansion, valid for large $N$, we can approximate the total energy of the particle 
configuration as

$$
\begin{aligned}
\mathcal{H}_{1}= & \frac{1}{2} N \epsilon_{1} \approx \frac{1}{2} N^{2}-\frac{1}{2} N^{3 / 2}+\frac{1}{12} N^{1 / 2}-\frac{1}{240} N^{-1 / 2} \\
& +\frac{1}{10080} N^{-3 / 2}-\frac{1}{725760} N^{-5 / 2}+\mathcal{O}\left(N^{-3}\right) .
\end{aligned}
$$

$$
\epsilon_{2}=\frac{N}{4 \pi} \int_{0}^{2 \pi} \int_{\theta_{0}}^{\pi} E_{1 i}^{(2)} \sin \theta d \theta d \phi, \quad E_{1 i}^{(2)}=-\frac{1}{2} \log r_{1 i}, \quad r_{1 i}=\sqrt{2(1-\cos \theta)}
$$

which can be evaluated explicitly to yield

$$
\varepsilon_{2}=-\log \left[\sin \left(N^{-1 / 2}\right)\right]\left(-\frac{N}{2}+\cos ^{2}\left(N^{-1 / 2}\right)\right)+(2 \log 2-1) \cos ^{2}\left(N^{-1 / 2}\right)
$$

From a Taylor series expansion, valid for $N \gg 1$, the total energy $\mathcal{H}_{2}=N \varepsilon_{2} / 2$ is estimated as

$$
\begin{aligned}
\mathcal{H}_{2} \approx & \frac{N^{2}}{8}(1-2 \log 2)-\frac{1}{8} N \log N-\frac{1}{8} N(1-2 \log 2)+\frac{1}{24} \log N-\frac{1}{12} \log 2 \\
& +N^{-1}\left(\frac{1}{144}+\frac{1}{90} \log 2\right)-\frac{1}{180} N^{-1} \log N-N^{-2}\left(\frac{7}{6480}-\frac{1}{1260} \log 2\right) \\
& +\frac{1}{2520} N^{-2} \log N+\mathcal{O}\left(N^{-3}\right) .
\end{aligned}
$$

The second logarithmic term $\mathcal{H}_{3}$ in $(4.2 \mathrm{~b})$ can be estimated in a similar way. We define $\epsilon_{3}$ as

$$
\epsilon_{3}=\frac{N}{4 \pi} \int_{0}^{2 \pi} \int_{\theta_{0}}^{\pi} E_{1 i}^{(3)} \sin \theta d \theta d \phi, \quad E_{1 i}^{(3)}=-\frac{1}{2} \log \left(2+r_{1 i}\right), \quad r_{1 i}=\sqrt{2(1-\cos \theta)} .
$$

We obtain analytically that

$$
\begin{gathered}
\epsilon_{3}=\frac{N}{4}\left[2 \sin \left(N^{-1 / 2}\right)-(2 \log 2-1) \cos ^{2}\left(N^{-1 / 2}\right)-2\right. \\
\left.-2 \cos ^{2}\left(N^{-1 / 2}\right) \log \left[1+\sin \left(N^{-1 / 2}\right)\right]\right] .
\end{gathered}
$$

For $N \gg 1$, the resulting total energy $\mathcal{H}_{3}=N \varepsilon_{3} / 2$ is estimated from a Taylor series expansion as

$$
\begin{aligned}
\mathcal{H}_{3} \approx- & \frac{N^{2}}{8}(1+2 \log 2)+\frac{\log 2}{4} N+\frac{1}{6} N^{1 / 2}-\left(\frac{1}{16}+\frac{1}{12} \log 2\right)-\frac{1}{20} N^{-1 / 2} \\
& +N^{-1}\left(\frac{1}{48}+\frac{1}{90} \log 2\right)+\frac{23}{5040} N^{-3 / 2}-N^{-2}\left(\frac{1}{480}+\frac{1}{1260} \log 2\right) \\
& -\frac{31}{90720} N^{-5 / 2}+\mathcal{O}\left(N^{-3}\right) .
\end{aligned}
$$

In Figure 4.5(a) we compare the sum $\mathcal{H}=\mathcal{H}_{1}+\mathcal{H}_{2}+\mathcal{H}_{3}$, obtained by adding (4.4), (4.7), and (4.10) (and neglecting the unspecified $\mathcal{O}\left(N^{-3}\right)$ terms), with the numerically

Copyright (c) by SIAM. Unauthorized reproduction of this article is prohibited. 


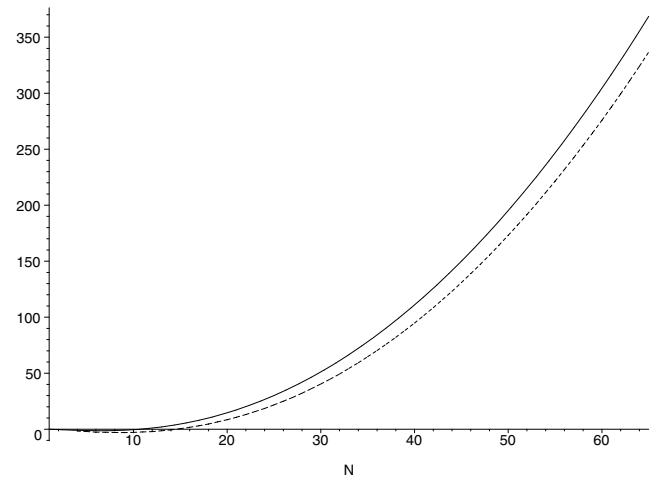

(a) Optimal $\mathcal{H}$ vs. $N$

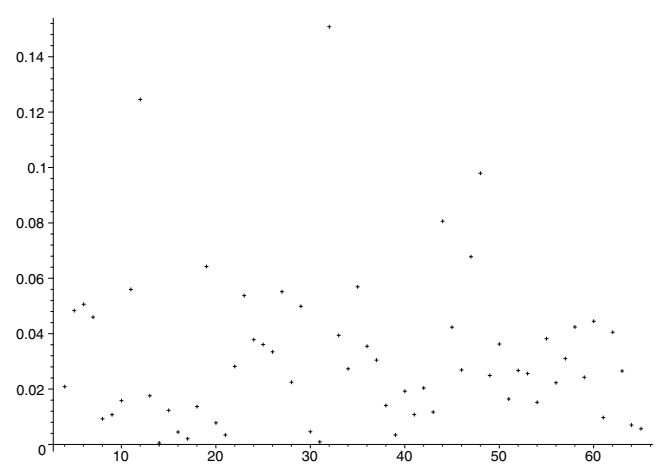

(b) Scatter Plot of the Error $|\mathcal{F}-\mathcal{H}|$ vs. $N$

FIG. 4.5. Top figure: comparison of full numerical results for the optimal energy $\mathcal{H}$ versus $N$ (dashed curve) with the large $N$ prediction given by the sum of (4.4), (4.7), and (4.10) (solid curve). Bottom figure: absolute value of the error between the full numerical results of Table 4.2 for $\mathcal{H}$ and the least squares fit $\mathcal{F}(N)$ of $\mathcal{H}$ given in (4.11).

computed results for the optimal values of $\mathcal{H}$ as given in Table 4.2. As seen in Figure $4.5(\mathrm{a})$, the agreement is relatively close.

The mean-field approximation completely disregards the spatial distribution of the optimal arrangement of particles on the sphere and therefore yields an approximate result whose precise asymptotic validity as $N \rightarrow \infty$ is very difficult to assess. The highest power of $N$ obtained by this approximation is presumably theoretically correct in analogy with previous rigorous results for the classical Coulomb or logarithmic energies (see [25], [33], and [34]). However, the coefficients of the lower-order terms should depend on the optimal trap arrangement. Therefore, in terms of some unknown coefficients $b_{j}$ for $j=1, \ldots, 6$, we postulate that for $N \gg 1$ the energy $\mathcal{H}$ has the form

$\mathcal{H} \approx \mathcal{F}(N)=\frac{N^{2}}{2}(1-\log 2)+b_{1} N^{3 / 2}+b_{2} N \log N+b_{3} N+b_{4} N^{1 / 2}+b_{5} \log N+b_{6}$,

as suggested by the various terms in (4.4), (4.7), and (4.10). The resulting least 
squares fit of (4.11a) to the data in Table 4.2 yields

$$
\begin{aligned}
& b_{1} \approx-0.5668, \quad b_{2} \approx 0.0628, \quad b_{3} \approx-0.8420, \\
& b_{4} \approx 3.8894, \quad b_{5} \approx-1.3512, \quad b_{6} \approx-2.4523 .
\end{aligned}
$$

In Figure 4.5(b) we show the scatter plot of the absolute error between $\mathcal{F}(N)$ and the data for $\mathcal{H}$ of Table 4.2. This figure indicates that $\mathcal{F}(N)$ provides a very good approximation to the numerically computed optimal values of $\mathcal{H}$.

Finally, by using the scaling law $\mathcal{H} \approx \frac{N^{2}}{2}(1-\log 2)+b_{1} N^{3 / 2}$ for large $N$, we obtain the following rough estimate of the minimum value of the average MFPT $\bar{v}$ in (2.51) for the case of $N \gg 1$ circular traps of a common radius $\varepsilon$ :

$$
\bar{v} \sim \frac{|\Omega|}{4 \varepsilon D N}\left[1-\frac{\varepsilon}{\pi} \log \varepsilon+\frac{\varepsilon N}{\pi}\left(\frac{1}{5}+\frac{4 b_{1}}{\sqrt{N}}\right)\right] .
$$

In terms of the trap surface area fraction $f$, given by $f=N \varepsilon^{2} / 4$, (4.12a) can be written equivalently as

$$
\bar{v} \sim \frac{|\Omega|}{8 D \sqrt{f N}}\left[1-\frac{\sqrt{f / N}}{\pi} \log \left(\frac{4 f}{N}\right)+\frac{2 \sqrt{f N}}{\pi}\left(\frac{1}{5}+\frac{4 b_{1}}{\sqrt{N}}\right)\right] .
$$

In order that the expansions $(4.12 \mathrm{a})$ and $(4.12 \mathrm{~b})$ remain ordered, we require that the third term in (4.12a) be asymptotically smaller than the second term. This enforces the requirement that $\varepsilon N \ll 1$ when $N \gg 1$. Therefore, (4.12b) holds for $N \gg 1$ when the trap area fraction satisfies $f \ll \mathcal{O}(\varepsilon)$. It would be very interesting to determine if there is a relationship between our optimal average MFPT results and corresponding results that can be obtained from the dilute trap volume fraction limit of homogenization theory.

5. Conclusion. The method of matched asymptotic expansions was used to calculate a three-term asymptotic expansion for the MFPT for escape from the unit sphere when there are $N$ locally circular traps of asymptotically small radii on the boundary of the unit sphere. The third term in this expansion was shown to depend on the spatial configuration of the traps on the surface of the sphere. For $N$ not too large, it was shown that the fragmentation of the trap set has a strong influence on the average MFPT for a fixed small trap surface area fraction.

There are four open problems that warrant further study. The first open problem is to give a rigorous justification of the three-term asymptotic result (2.44) derived here formally by the method of matched asymptotic expansions. The second open problem is to derive a result analogous to (2.44) for the average MFPT for an arbitrary bounded three-dimensional domain that has $N$ asymptotically small windows of radius $\mathcal{O}(\varepsilon)$ on its boundary. Only the third term in such an expansion should depend on the relative locations of the absorbing windows on the domain boundary. Such an analysis would require detailed knowledge of the regular part of the surface Neumann Green's function for an arbitrary three-dimensional domain. A two-term expansion for this MFPT, which is independent of the window locations, is given in [44] for an arbitrary bounded three-dimensional domain.

A third interesting open problem is to explore whether there is any relationship between results that can be obtained from the dilute trap fraction limit of homogenization theory and the results obtained herein for the scaling law for the discrete 
energy function for large $N$. Such an approach should hopefully provide an asymptotic formula for the average MFPT that is uniform in $\varepsilon$ and $N$. To illustrate the need for such a result, consider the narrow capture problem of [26] for a viral particle that has entered a biological cell containing a small spherical nucleus with 2000 nanopores on its surface. For this problem, the ratio of the radius of the nanopore to the radius of the nucleus is $\varepsilon=2 \times 10^{-3}$. Therefore, since $\varepsilon N \approx 4$, the limiting result (4.12a) is not valid, and an alternative approach based on homogenization theory is required to estimate the average MFPT.

Finally, a fourth open problem is to calculate a high-order asymptotic expansion for the average MPFT for the case of interior traps of small radii within a threedimensional domain, or to determine the average MFPT for some narrow capture problems. Some results in this direction are given in [5].

Appendix A. The surface Neumann Green's function for a sphere. Here we calculate the explicit solution to (2.2). Since the singular point is on the boundary of the sphere, then $G_{s}\left(x ; x_{j}\right) \sim(2 \pi)^{-1} /\left|x-x_{j}\right|$ as $x \rightarrow x_{j} \in \partial \Omega$. Our goal is to calculate $G_{s}\left(x ; x_{j}\right)$ analytically and to determine higher-order terms in the singular behavior as $x \rightarrow x_{j}$. To solve (2.2) analytically, we let $|x|^{2}=r^{2}$ and decompose $G_{s}$ as

$$
G_{s}=\frac{1}{6|\Omega|}\left(|x|^{2}+1\right)+\bar{G}_{s}+C,
$$

where $C$ is a constant chosen to ensure that $\int_{\Omega} G_{s} d x=0$. Then, we obtain from (A.1) and (2.2) that $\bar{G}_{s}$ satisfies

$$
\triangle \bar{G}_{s}=0, \quad x \in \Omega ;\left.\quad \partial_{r} \bar{G}_{s}\right|_{r=1}=\delta\left(\cos \theta-\cos \theta_{j}\right) \delta\left(\phi-\phi_{j}\right)-\frac{1}{4 \pi} .
$$

Next, the boundary condition in (A.2) is expressed in terms of Legendre polynomials. This is done as follows.

Lemma A.1. Let $\gamma$ denote the angle between $x$ and $x_{j}$ so that $\cos \gamma=x \cdot x_{j}$. Then, we have the identity

$$
\left.\partial_{r} \bar{G}_{s}\right|_{r=1}=\delta\left(\cos \theta-\cos \theta_{j}\right) \delta\left(\phi-\phi_{j}\right)-\frac{1}{4 \pi}=\frac{1}{4 \pi} \sum_{m=1}^{\infty}(2 m+1) P_{m}(\cos \gamma) .
$$

Proof. We recall the completeness formula for the spherical harmonics $Y_{m n}$ given by (cf. [22])

$$
\sum_{m=0}^{\infty} \sum_{n=-m}^{m} Y_{m n}^{*}\left(\theta_{j}, \phi_{j}\right) Y_{m n}(\theta, \phi)=\delta\left(\phi-\phi_{j}\right) \delta\left(\cos \theta-\cos \theta_{j}\right),
$$

where $*$ denotes complex conjugate. The well-known addition theorem for Legendre polynomials (cf. [22]) states that

$$
\frac{(2 m+1)}{4} P_{m}(\cos \gamma)=\sum_{n=-m}^{m} Y_{m n}^{*}\left(\theta_{j}, \phi_{j}\right) Y_{m n}(\theta, \phi) .
$$

Upon summing (A.5) from $m=0$ to $\infty$ and using (A.4) and $P_{0}(\cos \gamma)=1$, we obtain (A.3). 
The solution to $\triangle \bar{G}_{s}=0$, which satisfies the boundary condition in (A.3), is simply

(A.6)

$\bar{G}_{s}=\frac{1}{4 \pi} \sum_{m=1}^{\infty} \frac{(2 m+1)}{m} r^{m} P_{m}(\cos \gamma)=\frac{1}{2 \pi} \sum_{m=1}^{\infty} r^{m} P_{m}(\cos \gamma)+\frac{1}{4 \pi} \sum_{m=1}^{\infty} \frac{r^{m}}{m} P_{m}(\cos \gamma)$

We now calculate the two terms in (A.6) separately.

The well-known generating function $\left(1-2 x t+t^{2}\right)^{-1 / 2}=\sum_{m=0}^{\infty} P_{m}(x) t^{n}$ shows that the first term in (A.6) is

$$
\frac{1}{2 \pi} \sum_{m=1}^{\infty} r^{m} P_{m}(\cos \gamma)=\frac{1}{2 \pi} \frac{1}{\sqrt{r^{2}+1-2 r \cos \gamma}}-\frac{1}{2 \pi}=\frac{1}{2 \pi\left|x-x_{j}\right|}-\frac{1}{2 \pi} .
$$

Next, we define $I(r) \equiv \sum_{m=1}^{\infty} \frac{r^{m}}{m} P_{m}(\cos \gamma)$. By differentiating $I$ and then using the generating function, we get

$$
I^{\prime}(r)=\frac{1}{r} \sum_{m=1}^{\infty} r^{m} P_{m}(\cos \gamma)=\frac{1}{r}\left[\frac{1}{\sqrt{1-2 r \cos \gamma+r^{2}}}-1\right] .
$$

Since $I(0)=0$, we can integrate the equation above and then use $\left|x-x_{j}\right|=$ $\left(1+r^{2}-2 r \cos \gamma\right)^{1 / 2}$ to obtain

$$
\begin{aligned}
I(r) & =\sum_{m=1}^{\infty} \frac{r^{m}}{m} P_{m}(\cos \gamma)=\int_{0}^{r}\left(\frac{1}{s} \frac{1}{\sqrt{1-2 s \cos \gamma+s^{2}}}-\frac{1}{s}\right) d s \\
& =\log \left(\frac{2}{1-r \cos \gamma+\left|x-x_{j}\right|}\right),
\end{aligned}
$$

which determines the second term in (A.6). Then, substituting (A.9) and (A.7) into (A.6) and using (A.1), we can write $G_{s}$ up to an arbitrary constant $C$ as

$$
G_{s}\left(x ; x_{j}\right)=\frac{1}{8 \pi}\left(|x|^{2}+1\right)+\frac{1}{2 \pi\left|x-x_{j}\right|}+\frac{1}{4 \pi} \log \left(\frac{2}{1-r \cos \gamma+\left|x-x_{j}\right|}\right)+C .
$$

Finally, the integral condition in (2.2), written as $\int_{0}^{2 \pi} \int_{0}^{\pi} \int_{0}^{1} G_{s} r^{2} \sin \gamma d r d \gamma d \phi=$ 0 , determines $C$. Here, without loss of generality, we have chosen $x_{j}$ to be at the north pole so that $\gamma=\theta$. By orthogonality of the $P_{m}(z)$, it follows that $\int_{0}^{\pi} P_{m}(\cos \gamma) \sin \gamma d \gamma$ $=2 \delta_{m, 0}$, where $\delta_{m, 0}$ is Kronecker's symbol, $\delta_{n, n}=1$, and $\delta_{m, n}=0$ if $m \neq n$. Therefore, this identity together with (A.9) shows that the integral over the sphere of the logarithmic term in (A.10) vanishes identically. Next, from (A.7) and $P_{0}(z)=1$, we use the same identity to calculate

$$
\frac{1}{2 \pi} \int_{\Omega} \frac{1}{\left|x-x_{j}\right|} d x=\frac{1}{2 \pi} \sum_{m=0}^{\infty} \int_{0}^{2 \pi} \int_{0}^{\pi} \int_{0}^{1} r^{m} P_{m}(\cos \gamma)(\sin \gamma) r^{2} d \gamma d \phi d r=\frac{2}{3} .
$$

In this way, we obtain that

$$
\int_{\Omega} G_{s} d x=\int_{0}^{2 \pi} \int_{0}^{\pi} \int_{0}^{1} G_{s} r^{2} \sin \gamma d \gamma d \phi d r=\frac{1}{2} \int_{0}^{1}\left(r^{2}+1\right) r^{2} d r+\frac{2}{3}+\frac{4 \pi C}{3}=0,
$$


which yields $C=-7 /(10 \pi)$ and determines $G_{s}$ explicitly in (A.10). This completes the proof of Lemma 2.1.

Appendix B. The inner problem for $\boldsymbol{w}_{2}$. Here we first derive the PDE (2.13) for the second inner correction term $w_{2}$. Under the mapping $\eta=\varepsilon^{-1}(1-r)$, $s_{1}=\varepsilon^{-1} \sin \theta_{j}\left(\phi-\phi_{j}\right)$, and $s_{2}=\varepsilon^{-1}\left(\theta-\theta_{j}\right)$, we show that the Poisson equation (2.1a) in spherical coordinates for $v(r, \phi, \theta)$ transforms for $\varepsilon \rightarrow 0$ to (2.13). Let $w\left(\eta, s_{1}, s_{2}\right)=v\left[r(\eta), \phi\left(s_{1}\right), \theta\left(s_{2}\right)\right]$. Then, we calculate

$$
\begin{aligned}
v_{r r}+\frac{2}{r} v_{r} & =\varepsilon^{-2} w_{\eta \eta}-2 \varepsilon^{-1} w_{\eta}+\mathcal{O}(1), \\
r^{-2}\left(v_{\theta \theta}+v_{\theta} \cot \theta\right) & =(1-\varepsilon \eta)^{-2}\left(\varepsilon^{-2} w_{s_{2} s_{2}}+\varepsilon^{-1} \cot \theta_{j} w_{s_{2}}+\mathcal{O}(1)\right) \\
& =\varepsilon^{-2} w_{s_{2} s_{2}}+\varepsilon^{-1}\left(2 \eta w_{s_{2} s_{2}}+\cot \theta_{j} w_{s_{2}}\right)+\mathcal{O}(1), \\
\frac{1}{r^{2} \sin ^{2} \theta} v_{\phi \phi} & =\frac{(1-\varepsilon \eta)^{-2}}{\left[\sin \left(\theta_{j}+\varepsilon s_{2}\right)\right]^{2}} \varepsilon^{-2} \sin ^{2} \theta_{j} w_{s_{1} s_{1}} \\
& =\varepsilon^{-2} w_{s_{1} s_{1}}+\varepsilon^{-1}\left[2 \eta w_{s_{1} s_{1}}-2 s_{2} \cot \theta_{j} w_{s_{1} s_{1}}\right]+\mathcal{O}(1) .
\end{aligned}
$$

Therefore, upon adding the three expressions above, we get that $\Delta v=-D^{-1}$ in (2.1a) becomes

$$
\begin{aligned}
\Delta v= & \varepsilon^{-2}\left(w_{\eta \eta}+w_{s_{1} s_{1}}+w_{s_{2} s_{2}}\right) \\
& +\varepsilon^{-1}\left[2 \eta\left(w_{s_{1} s_{1}}+w_{s_{2} s_{2}}\right)-2 w_{\eta}+\cot \theta_{j}\left(w_{s_{2}}-2 s_{2} w_{s_{1} s_{1}}\right)\right] \\
& +\mathcal{O}(1)=-\frac{1}{D} .
\end{aligned}
$$

If we then expand $w \sim \varepsilon^{-1} w_{0}+\log \left(\frac{\varepsilon}{2}\right) w_{1}+w_{2}+\cdots$ as in (2.12), we readily obtain (2.13) for $w_{2}$ upon using the leading-order equation $w_{0 \eta \eta}=-\left(w_{0 s_{1} s_{1}}+w_{0 s_{2} s_{2}}\right)$ to simplify the coefficient of $\eta$ in (B.1).

Next, we analyze the solution $w_{2}$ to (2.13), with the prescribed far-field behavior in (2.31), in terms of the solution decomposition given in (2.32). Our analysis shows how the solution to this problem generates a monopole term in its far-field expansion as written in (2.35), where the monopole coefficient $\kappa_{j}$ in (2.35) is as given in (2.36).

We analyze (2.33) for the term $w_{2 e}$ in the decomposition (2.32) of $w_{2}$. Although (2.33) is an inhomogeneous problem involving the solution $w_{c}$ of the electrified disk problem (2.15), its solution $w_{2}$ can be determined analytically.

Lemma B.1. The solution to (2.33) is given explicitly by

$$
w_{2 e}=-\frac{\eta^{2}}{2} w_{c \eta}-\frac{\eta}{2} w_{c}+\frac{1}{2} \int_{0}^{\eta} w_{c}\left(z, s_{1}, s_{2}\right) d z+\mathcal{K}\left(s_{1}, s_{2}\right)+w_{2 h} .
$$

Here $\mathcal{K}\left(s_{1}, s_{2}\right)$ satisfies a Poisson equation with a compactly supported forcing function, formulated as

(B.3a)

$\mathcal{K}_{s_{1} s_{1}}+\mathcal{K}_{s_{2} s_{2}}=q\left(s_{1}, s_{2}\right) ; \quad \mathcal{K}\left(s_{1}, s_{2}\right)=\frac{c_{j}}{2} \log \sigma+o(1) \quad$ as $\quad \sigma \equiv\left(s_{1}^{2}+s_{2}^{2}\right)^{1 / 2} \rightarrow \infty$,

where $c_{j}$ is defined by $w_{c} \sim c_{j} / \rho$ as $\rho \rightarrow \infty$, while $q\left(s_{1}, s_{2}\right)$ is defined in terms of the surface charge density of the electrified disk problem (2.15) by

$$
q\left(s_{1}, s_{2}\right) \equiv-\left.\frac{1}{2} w_{c \eta}\right|_{\eta=0} I_{\Omega}, \quad I_{\Omega}= \begin{cases}1, & \left(s_{1}, s_{2}\right) \in \Omega, \\ 0, & \left(s_{1}, s_{2}\right) \notin \Omega,\end{cases}
$$

Copyright (c) by SIAM. Unauthorized reproduction of this article is prohibited. 
where $\Omega \equiv\left\{\left(s_{1}, s_{2}\right) \mid s_{1}^{2}+s_{2}^{2} \leq a_{j}^{2}\right\}$. Moreover, in terms of $\mathcal{K}\left(s_{1}, s_{2}\right)$, the function $w_{2 h}$ in (B.2) satisfies Laplace's equation in a half-space with mixed Dirichlet-Neumann boundary conditions, formulated as

$$
\partial_{\eta} w_{2 h}=0, \quad \eta=0,\left(s_{1}, s_{2}\right) \notin \Omega ; \quad w_{2 h}=-\mathcal{K}\left(s_{1}, s_{2}\right), \quad \eta=0,\left(s_{1}, s_{2}\right) \in \Omega,
$$

$$
w_{2 h}=\mathcal{O}\left(\rho^{-1}\right) \quad \text { as } \quad \rho=\left(\eta^{2}+s_{1}^{2}+s_{2}^{2}\right)^{1 / 2} \rightarrow \infty .
$$

The solution $w_{2 e}$ in (B.2) has the far-field asymptotic behavior

$$
w_{2 e}=\frac{c_{j}}{2} \log (\eta+\rho)-\frac{c_{j}}{2 \rho^{3}} \eta\left(s_{1}^{2}+s_{2}^{2}\right)-\frac{c_{j} \kappa_{j}}{\rho}+\mathcal{O}\left(\rho^{-2}\right) \quad \text { as } \quad \rho \rightarrow \infty .
$$

We remark that the $o(1)$ condition in (B.3a) and the decay condition in (B.4c) determine $\mathcal{K}\left(s_{1}, s_{2}\right)$ and $w_{2 h}$ uniquely. In addition, it is readily observed that the problem for $\mathcal{K}$ has the correct strength for the logarithmic singularity at infinity. To see this, we let $s=\left(s_{1}, s_{2}\right)$ and solve (B.3) in terms of the free-space Green's function as

$$
\begin{aligned}
\mathcal{K}\left(s_{1}, s_{2}\right)= & \frac{1}{2 \pi} \int_{\Omega} \log |\tilde{s}-s| q(\tilde{s}) d s \sim\left(\frac{1}{2 \pi} \int_{\Omega} q(\tilde{s}) d \tilde{s}\right) \log \sigma \\
& -\frac{s \cdot e_{j}}{2 \pi|s|^{2}}+\mathcal{O}\left(|s|^{-2}\right) \quad \text { as } \quad \sigma=|s| \rightarrow \infty,
\end{aligned}
$$

where $e_{j} \equiv \int_{\Omega} \tilde{s}_{j} q(\tilde{s}) d \tilde{s}$. Therefore, $\mathcal{K} \sim d_{0} \log \sigma+o(1)$ as $\sigma \rightarrow \infty$, where $d_{0}=$ $-\left.(4 \pi)^{-1} \int_{\Omega} w_{c \eta}\right|_{\eta=0} d \tilde{s}$. To identify that $d_{0}=c_{j} / 2$, where $c_{j}$ is the monopole coefficient obtained from the far-field behavior $w_{c} \sim c_{j} / \rho$ as $\rho \rightarrow \infty$, we use the divergence theorem over a half-sphere on the defining problem (2.15) for $w_{c}$ to obtain $-2 \pi c_{j}+$ $\int_{\Omega}\left(-\left.w_{c \eta}\right|_{\eta=0}\right) d \tilde{s}=0$. This yields $c_{j}=-\left.(2 \pi)^{-1} \int_{\Omega} w_{c \eta}\right|_{\eta=0} d \tilde{s}$, so that $d_{0}=c_{j} / 2$ as required.

There are three main steps to the proof of Lemma B.1. First, we must show that (B.2) accounts for the inhomogeneous term in the PDE (2.33a). Then, we must show that the boundary conditions (2.33b) and the far-field behavior (2.33c) are both satisfied.

By an explicit calculation we first verify that (B.2) satisfies the PDE (2.33a). Since $w_{2 h}$ satisfies the homogeneous problem, we need only verify that $w_{2 p}$, defined by

$$
w_{2 p} \equiv-\frac{\eta^{2}}{2} w_{c \eta}-\frac{\eta}{2} w_{c}+\frac{1}{2} \int_{0}^{\eta} w_{c}\left(z, s_{1}, s_{2}\right) d z+\mathcal{K}\left(s_{1}, s_{2}\right),
$$

satisfies (2.33a). Denoting $\mathcal{L} v \equiv v_{\eta \eta}+\Delta_{s} v$, where $\Delta_{s} v \equiv v_{s_{1} s_{1}}+v_{s_{2} s_{2}}$, we calculate

$$
\begin{gathered}
w_{2 p \eta \eta}=-\frac{\eta^{2}}{2} w_{c \eta \eta \eta}-\frac{5 \eta}{2} w_{c \eta \eta}-\frac{3}{2} w_{c \eta}, \\
\Delta_{s} w_{2 p}=-\frac{\eta^{2}}{2} \partial_{\eta}\left(\Delta_{s} w_{c}\right)-\frac{\eta}{2} \Delta_{s} w_{c}+\frac{1}{2} \int_{0}^{\eta} \Delta_{s} w_{c} d z+\Delta_{s} \mathcal{K} .
\end{gathered}
$$

Then, using the equation $w_{c \eta \eta}=-\Delta_{s} w_{c}$ satisfied by $w_{c}$, (B.8b) becomes

$$
\begin{aligned}
\Delta_{s} w_{2 p} & =\frac{\eta^{2}}{2} w_{c \eta \eta \eta}+\frac{\eta}{2} w_{c \eta \eta}-\frac{1}{2} \int_{0}^{\eta} w_{c z z} d z+\Delta_{s} \mathcal{K} \\
& =\frac{\eta^{2}}{2} w_{c \eta \eta \eta}+\frac{\eta}{2} w_{c \eta \eta}-\frac{1}{2} w_{c \eta}+\left.\frac{1}{2} w_{c \eta}\right|_{\eta=0}+\Delta_{s} \mathcal{K} .
\end{aligned}
$$

Copyright $@$ by SIAM. Unauthorized reproduction of this article is prohibited. 
Upon adding (B.8a) and (B.9), we calculate $\mathcal{L} w_{2 p}=w_{2 p \eta \eta}+\Delta_{s} w_{2 p}$ as

$$
\mathcal{L} w_{2 p}=-2 \eta w_{c \eta \eta}-2 w_{c \eta}+\left.\frac{1}{2} w_{c \eta}\right|_{\eta=0}+\Delta_{s} \mathcal{K}\left(s_{1}, s_{2}\right) .
$$

Therefore, when $\mathcal{K}\left(s_{1}, s_{2}\right)$ satisfies the Poisson equation (B.3), it follows that $w_{2 p}$ satisfies the PDE (2.33a).

Next, we note from (B.2) that on $\eta=0$ the boundary conditions $\partial_{\eta} w_{2 e}=0$ for $\left(s_{1}, s_{2}\right) \notin \Omega$ and $w_{2 e}=0$ for $\left(s_{1}, s_{2}\right) \in \Omega$ are satisfied provided that $w_{2 h}$ satisfies the boundary conditions in (B.4b).

Next, we determine the asymptotic far-field behavior of $w_{2 e}$ as defined in (B.2). We use $w_{c} \sim c_{j} \rho^{-1}$ as $\rho \rightarrow \infty$ with $\rho=\left(\eta^{2}+\sigma^{2}\right)^{1 / 2}$ and $\sigma=\left(s_{1}^{2}+s_{2}^{2}\right)^{1 / 2}$ to calculate

$$
\begin{aligned}
& -\frac{1}{2} \eta^{2} w_{c \eta}-\frac{1}{2} \eta w_{c} \sim-\frac{c_{j}}{2} \frac{\eta \sigma^{2}}{\left(\eta^{2}+\sigma^{2}\right)^{3 / 2}} \quad \text { as } \quad \rho \rightarrow \infty \\
& \frac{1}{2} \int_{0}^{\eta} w_{c}\left(z, s_{1}, s_{2}\right) d z \sim \frac{c_{j}}{2} \int_{0}^{\eta} \frac{1}{\left(z^{2}+\sigma^{2}\right)^{1 / 2}} d z \\
& \quad=\frac{c_{j}}{2}\left[\log \left(\eta+\sqrt{\eta^{2}+\sigma^{2}}\right)-\log \sigma\right] \quad \text { as } \quad \rho \rightarrow \infty .
\end{aligned}
$$

Therefore, $w_{2 e}$ in (B.2) has the far-field behavior

$$
w_{2 e} \sim \frac{c_{j}}{2} \log \left(\eta+\sqrt{\eta^{2}+\sigma^{2}}\right)-\frac{c_{j}}{2} \frac{\eta \sigma^{2}}{\left(\eta^{2}+\sigma^{2}\right)^{3 / 2}}-\frac{c_{j}}{2} \log \sigma+\mathcal{K}+w_{2 h} .
$$

We observe that the first two terms on the right-hand side of (B.12) agree exactly with those in (2.33c). In order that the remaining terms in the far-field behavior (B.12) cancel, as indicated by (2.33c), we require that $\mathcal{K} \sim \frac{c_{j}}{2} \log \sigma+o(1)$ as $\sigma \rightarrow \infty$, as written in (B.3a), and that $w_{2 h} \rightarrow 0$ as $\rho \rightarrow \infty$, as given in (B.4c).

The problem (B.4) for $w_{2 h}$ is a mixed Dirichlet-Neumann boundary value problem for the Laplacian with a spatially inhomogeneous Dirichlet condition imposed on the absorbing window. As such, it follows that $w_{2 h}=\mathcal{O}\left(\rho^{-1}\right)$ as $\rho \rightarrow \infty$. Moreover, since $\mathcal{K}$ is proportional to $c_{j}$, we can write the far-field behavior for $w_{2 h}$ as

$$
w_{2 h}=-\frac{c_{j} \kappa_{j}}{\rho}+\mathcal{O}\left(\rho^{-2}\right) \quad \text { as } \quad \rho \rightarrow \infty
$$

for some monopole coefficient $\kappa_{j}$ to be determined.

We remark that up to this stage of the analysis in this appendix we have not assumed that the absorbing window $\Omega$ is a circular disk of radius $a_{j}$. All that has been required so far is that $w_{c}$ satisfy $\mathcal{L} w_{c}=0$ with boundary conditions $\partial_{n} w_{c}=0$ on $\eta=0,\left(s_{1}, s_{2}\right) \notin \Omega$ and $w_{c}=1$ on $\eta=0$, and $\left(s_{1}, s_{2}\right) \in \Omega$ with $w_{c} \sim c_{j} / \rho$ as $\rho \rightarrow \infty$.

For the special case of a circular absorbing window of radius $a_{j}$, we can solve (B.3) analytically and then explicitly calculate the coefficient $\kappa_{j}$ of the monopole term in (B.13). For a circular disk, the function $q$ in (B.3) is simply $q\left(s_{1}, s_{2}\right)=$ $\pi^{-1}\left[a_{j}^{2}-\sigma^{2}\right]^{-1 / 2}$, where $\sigma=\left(s_{1}^{2}+s_{2}^{2}\right)^{1 / 2}$ (see page 38 of [12]). Therefore, from (B.3), $\mathcal{K}=\mathcal{K}(\sigma)$ is the solution of the radially symmetric problem

$$
\sigma^{-1}\left(\sigma \mathcal{K}_{\sigma}\right)_{\sigma}= \begin{cases}\pi^{-1}\left[a_{j}^{2}-\sigma^{2}\right]^{-1 / 2}, & 0 \leq \sigma<a_{j}, \\ 0, & \sigma \geq a_{j},\end{cases}
$$

Copyright $@$ ( ) by SIAM. Unauthorized reproduction of this article is prohibited. 
with $\mathcal{K}(\sigma)=a_{j} \pi^{-1} \log \sigma$ for $\sigma \geq a_{j}$. The solution to (B.14) for $\sigma \leq a_{j}$ with $\mathcal{K}\left(a_{j}\right)=$ $a_{j} \pi^{-1} \log \left(a_{j}\right)$ is simply

$$
\mathcal{K}(\sigma)=\frac{1}{\pi}\left[a_{j} \log \left(a_{j}+\sqrt{a_{j}^{2}-\sigma^{2}}\right)-\sqrt{a_{j}^{2}-\sigma^{2}}\right], \quad 0 \leq \sigma \leq a_{j} .
$$

For a circular disk, the coefficient $\kappa_{j}$ of the monopole term in (B.13) can be calculated by using the known far-field behavior of $w_{2 h}$ given by (see [10] or section 1.4 of [12])

$$
w_{2 h} \sim-\frac{2}{\pi \rho} \int_{0}^{a_{j}} \frac{\mathcal{K}(\sigma) \sigma}{\sqrt{a_{j}^{2}-\sigma^{2}}} d \sigma \quad \text { as } \quad \rho \rightarrow \infty .
$$

The integral in (B.16) can be calculated explicitly by using (B.15) for $\mathcal{K}(\sigma)$. This yields

$$
\begin{gathered}
J \equiv \int_{0}^{a_{j}} \frac{\mathcal{K}(\sigma) \sigma}{\sqrt{a_{j}^{2}-\sigma^{2}}} d \sigma=\frac{a_{j}^{2}}{\pi}\left[\int_{0}^{1} \frac{x}{\sqrt{1-x^{2}}}\left(\log \left[1+\sqrt{1-x^{2}}\right]-\sqrt{1-x^{2}}\right) d x\right. \\
\left.+\log a_{j} \int_{0}^{1} \frac{x}{\sqrt{1-x^{2}}} d x\right]
\end{gathered}
$$

which evaluates to $J=a_{j}^{2} \pi^{-1}\left[2 \log 2-\frac{3}{2}+\log a_{j}\right]$. Since $c_{j}=2 a_{j} / \pi$, it follows from (B.13) and (B.16) that

$$
w_{2 h} \sim-\frac{c_{j} \kappa_{j}}{\rho}, \quad \rho \rightarrow \infty, \quad \kappa_{j}=\frac{c_{j}}{2}\left[2 \log 2-\frac{3}{2}+\log a_{j}\right] .
$$

This detailed analysis of the solution to the inner problem for $w_{2}$ completes the proof of Lemma B.1 and justifies the far-field result (2.37) with $\kappa_{j}$ as given in (2.36).

Finally, we remark that the solution $w_{2 o}$ to (2.34) is odd in $s_{2}$ and therefore cannot generate a monopole term at infinity. The explicit solution to (2.34) is given in terms of the solution $w_{c}$ of the electrified disk problem (2.15) as follows.

Lemma B.2. The solution to (2.34) is given explicitly by

$$
w_{2 o}=\cot \theta_{j}\left(\frac{s_{1}^{2}}{2} w_{c s_{2}}-s_{2} s_{1} w_{c s_{1}}\right) .
$$

It is readily verified that (B.19) incorporates the inhomogeneous terms in (2.34a), satisfies the boundary conditions in (2.34b), and has far-field asymptotic behavior that agrees with that in $(2.34 \mathrm{c})$. We leave the verification of these details to the reader.

Acknowledgments. We would like to thank Sheldon Richards and Professor Raymond Spiteri of the Department of Computer Science at the University of Saskatchewan for providing Table 4.2. Also, we are grateful to a referee for a few very helpful comments.

\section{REFERENCES}

[1] G. Beliakov, The cutting angle method - a tool for constrained global optimization, Optim. Methods Softw., 19 (2004), pp. 137-151.

Copyright (c) by SIAM. Unauthorized reproduction of this article is prohibited. 
[2] O. BÉnichou And R. Voituriez, Narrow escape time problem: Time needed for a particle to exit a confining domain through a small window, Phys. Rev. Lett., 100 (2008), 168105.

[3] B. Bergersen, D. Boal, and P. Palffyy-Muhoray, Equilibrium configurations of particles on the sphere: The case of logarithmic interactions, J. Phys. A, 27 (1994), pp. 2579-2586.

[4] A. Burchard And J. Denzler, On the geometry of optimal windows, with special focus on the square, SIAM J. Math. Anal., 37 (2006), pp. 1800-1827.

[5] A. Cheviakov and M. J. Ward, Optimizing the fundamental eigenvalue of the Laplacian in a sphere with interior traps, Math. Comput. Modelling, to appear.

[6] COMSOL Multiphysics (v3.4), COMSOL AB, Stockholm, Sweden.

[7] D. Coombs, R. Straube, and M. Ward, Diffusion on a sphere with localized traps: Mean first passage time, eigenvalue asymptotics, and Fekete points, SIAM J. Appl. Math., 70 (2009), pp. 302-332.

[8] A. M. Davis and S. G. Llewellyn Smith, Perturbation of eigenvalues due to gaps in twodimensional boundaries, Proc. R. Soc. Lond. Ser. A Math. Phys. Eng. Sci., 463 (2007), pp. 759-786.

[9] J. Denzler, Windows of a given area with minimal heat diffusion, Trans. Amer. Math. Soc., 351 (1999), pp. 569-680.

[10] A. B. Efimov And V. N. Vorobev, A mixed boundary-value problem for the Laplace equation, J. Eng. Phys. Thermophys., 26 (1974), pp. 664-666.

[11] T. Erber and G. M. Hockney, Equilibrium configurations of $N$ equal charges on a sphere, J. Phys. A, 24 (1991), pp. 1369-1377.

[12] V. I. Fabrikant, Applications of Potential Theory to Mechanics: A Selection of New Results, Math. Appl. 51, Kluwer Academic Publishers, Dordrecht, The Netherlands, 1989.

[13] V. I. Fabrikant, Potential of several arbitrarily located disks, J. Austral. Math. Soc. Ser. B, 29 (1988), pp. 342-351.

[14] GANSO Software Library, University of Ballarat, Ballarat, Victoria, Australia, http://www. ballarat.edu.au/ciao.

[15] L. Glasser And A. G. Every, Energies and spacings of point charges on a sphere, J. Phys. A, 25 (1992), pp. 2473-2482.

[16] I. V. Grigoriev, Y. A. Makhnovskit, A. M. Berezhrovskit, and V. Y. Zitserman, Kinetics of escape through a small hole, J. Chem. Phys., 116 (2002), pp. 9574-9577.

[17] D. P. Hardin And E. B. SAFF, Discretizing manifolds via minimum energy points, Notices Amer. Math. Soc., 51 (2004), pp. 1186-1194.

[18] R. H. Hardin, N. J. A. Sloane, and W. D. Smith, Minimal Energy Arrangements of Points on a Sphere, http://www.research.att.com/ njas/electrons/index.html.

[19] D. Holcman And Z. Schuss, Escape through a small opening: Receptor trafficking in a synaptic membrane, J. Stat. Phys., 117 (2004), pp. 975-1014.

[20] D. Holcman And Z. Schuss, Diffusion escape through a cluster of small absorbing windows, J. Phys. A, 41 (2008), 155001.

[21] R. Horst and P. M. Pardalos, eds., Handbook of Global Optimization, Kluwer Academic Publishers, Dordrecht, Boston, 1995.

[22] J. D. Jackson, Classical Electrodynamics, 2nd ed., Wiley, New York, 1945.

[23] O. D. Kellog, Foundations of Potential Theory, Dover, New York, 1954.

[24] T. Kolokolnikov, M. Titcombe, And M. J. Ward, Optimizing the fundamental Neumann eigenvalue for the Laplacian in a domain with small traps, European J. Appl. Math., 16 (2005), pp. 161-200.

[25] A. B. J. Kuijlaars and E. B. SAFF, Asymptotics for minimal discrete energy on the sphere, Trans. Amer. Math. Soc., 350 (1998), pp. 523-538.

[26] T. Lagache, E. Dauty, And D. Holcman, Quantitative analysis of virus and plasmid trafficking in cells, Phys. Rev. E (3), 79 (2009), 011921.

[27] P. Lagerstrom, Matched Asymptotic Expansions: Ideas and Techniques, Appl. Math. Sci. 76, Springer-Verlag, New York, 1988.

[28] P. A. Lagerstrom and D. A. Reinelt, Note on logarithmic switchback terms in regular and singular perturbation expansions, SIAM J. Appl. Math., 44 (1984), pp. 451-462.

[29] M. A. Mammadov, A. Rubinov, and J. Yearwood, Dynamical systems described by relational elasticities with applications to global optimization, in Continuous Optimization: Current Trends and Modern Applications, A. Rubinov and V. Jeyakumar, eds., Springer-Verlag, New York, 2005, pp. 365-385.

[30] S. Pillay, M. J. Ward, A. Peirce, And T. Kolokolnikov, An asymptotic analysis of the mean first passage time for narrow escape problems: Part I: Two-dimensional domains, Multiscale Model. Simul., 8 (2010), pp. 803-835.

[31] J. D. PIntér, Global Optimization in Action, Kluwer Academic Publishers, Dordrecht, The Netherlands, 1996.

Copyright (c) by SIAM. Unauthorized reproduction of this article is prohibited. 
[32] I. Y. Popov, Extension theory and localization of resonances for domains of trap type, Math. USSR-Sb., 71 (1992), pp. 209-234.

[33] E. A. Rakhmanov, E. B. Saff, And Y. M. Zhou, Minimal discrete energy on the sphere, Math. Res. Lett., 1 (1994), pp. 647-662.

[34] E. A. Rakhmanov, E. B. Saff, and Y. M. Zhou, Electrons on the sphere, in Computational Methods and Function Theory 1994 (Penang), Ser. Approx. Decompos. 5, World Scientific, River Edge, NJ, 1995, pp. 293-309.

[35] S. Redner, A Guide to First-Passage Time Processes, Cambridge University Press, Cambridge, UK, 2001.

[36] A. Rubinov and V. Jeyakumar, eds., Continuous Optimization: Current Trends and Modern Applications, Springer-Verlag, New York, 2005.

[37] A. N. Rukhovets and Y. S. Uflyand, Electrostatic field of a system of non-parallel circular disks, Sov. Phys. Tech. Phys., 41 (1971), pp. 1103-1108.

[38] Z. Schuss, Theory and Applications of Stochastic Differential Equations, Wiley Ser. Probab. Stat., John Wiley \& Sons, New York, 1980.

[39] Z. Schuss, A. Singer, And D. Holcman, The narrow escape problem for diffusion in cellular microdomains, Proc. Natl. Acad. Sci. USA, 104 (2007), pp. 16098-16103.

[40] A. S. Silbergleit, I. Mandel, and I. M. Nemenman, Potential and field singularity at a surface point charge, J. Math. Phys., 44 (2003), pp. 4460-4466.

[41] A. Singer, Z. Schuss, D. Holcman, and R. S. Eisenberg, Narrow escape, Part I, J. Stat. Phys., 122 (2006), pp. 437-463.

[42] A. Singer, Z. Schuss, and D. Holcman, Narrow escape, Part II: The circular disk, J. Stat. Phys., 122 (2006), pp. 465-489.

[43] A. Singer, Z. Schuss, and D. Holcman, Narrow escape, Part III: Non-smooth domains and Riemann surfaces, J. Stat. Phys., 122 (2006), pp. 491-509.

[44] A. Singer, Z. Schuss, And D. Holcman, Narrow escape and leakage of Brownian particles, Phys. Rev. E (3), 78 (2008), 051111.

[45] R. SPITERI, private communication.

[46] J. Tausch And J. White, Second-kind integral formulations of the capacitance problem, Adv. Comput. Math., 9 (1998), pp. 217-232.

[47] M. J. WARD AND J. B. KelLeR, Strong localized perturbations of eigenvalue problems, SIAM J. Appl. Math., 53 (1993), pp. 770-798.

Copyright $@$ by SIAM. Unauthorized reproduction of this article is prohibited. 